\title{
Vector-Quantization-Based Topic Modeling
}

\author{
AMULYA GUPTA and ZHU ZHANG, Iowa State University
}

\begin{abstract}
With the purpose of learning and utilizing explicit and dense topic embeddings, we propose three variations of novel vector-quantization-based topic models (VQ-TMs): (1) Hard VQ-TM, (2) Soft VQ-TM, and (3) Multi-View Soft VQ-TM. The model family capitalize on vector quantization techniques, embedded input documents, and viewing words as mixtures of topics. Guided by a comprehensive set of evaluation metrics, we conduct systematic quantitative and qualitative empirical studies, and demonstrate the superior performance of VQTMs compared to important baseline models. Through a unique case study on code generation from natural language descriptions, we further illustrate the power of VQ-TMs in downstream tasks.
\end{abstract}

CCS Concepts: • Information systems $\rightarrow$ Information retrieval; Document representation; Document topic models; $\bullet$ Software and its engineering $\rightarrow$ Software creation and management; Software development techniques;

Additional Key Words and Phrases: Knowledge discovery, deep learning, self-supervised learning

\section{ACM Reference format:}

Amulya Gupta and Zhu Zhang. 2021. Vector-Quantization-Based Topic Modeling. ACM Trans. Intell. Syst. Technol. 12, 3, Article 34 (April 2021), 30 pages.

https://doi.org/10.1145/3450946

\section{INTRODUCTION}

Representation learning is fundamental to any artificial intelligence (AI) system [3]. Specifically, the system should be able to learn the underlying explanatory factors hidden in the observed data that may have utilities in various downstream tasks. Such latent factors take different forms depending on the domain under consideration. For instance, Paccanaro and Hinton [28] consider the set of operations among integers as latent concepts that in turn are represented as $n$-dimensional vectors. Similarly, Higgens et al. [16] illustrate the importance of learning basic visual latent concepts such as azimuth from multiple datasets in computer vision. In a different domain of modeling interaction between natural language (NL) and code snippets, Neubig and Allamanis [26] define coding as implementation of hidden concepts.

In natural language processing and information retrieval, topic modeling aims at extracting latent topics or concepts from a set of documents. The year 1988 saw the seminal work on latent semantic indexing [7], which is among the earliest to reveal the latent semantic structure present in a document collection. Later, probabilistic models such as Latent Dirichlet Allocation (LDA) [5] and hierarchical Dirichlet processes [36] have played significant roles in topic modeling. A common characteristic of these traditional models is viewing topics as sparse distributions over

Authors' address: A. Gupta, 2434 Osborn Dr, Ames, IA 50011-1090; email: guptaam@iastate.edu; Z. Zhang, 2167 Union Drive, Ames, IA 50011-2027; email: zhuzhang@iastate.edu.

Permission to make digital or hard copies of all or part of this work for personal or classroom use is granted without fee provided that copies are not made or distributed for profit or commercial advantage and that copies bear this notice and the full citation on the first page. Copyrights for components of this work owned by others than ACM must be honored. Abstracting with credit is permitted. To copy otherwise, or republish, to post on servers or to redistribute to lists, requires prior specific permission and/or a fee. Request permissions from permissions@acm.org.

(C) 2021 Association for Computing Machinery.

2157-6904/2021/04-ART34 \$15.00

https://doi.org/10.1145/3450946 
words, which only works well with bag-of-words (BOW)-based feature representations for text. The rise of deep learning as a new paradigm calls for topic representations more coherent with neural architectures. Therefore, inspired by related work reviewed in Section 2, we aim at the following:

(1) Learning explicit dense topic embeddings homomorphic to word embeddings,

(2) Utilizing learned topic embeddings in downstream tasks.

With these goals in mind, we propose novel vector-quantization-based neural models capable of learning latent topics embedded in the same semantic space as words. We empirically demonstrate the benefits of

(1) Vector quantization for learning discrete latent representations,

(2) Taking advantage of word-embedding-based document representations, and

(3) Directly modeling words as mixtures of topics.

Finally, we illustrate the utilization of learned latent topics/concepts through a case study in code generation from English descriptions.

\section{RELATED WORK}

\subsection{Topic Modeling Overview}

Recent work in topic modeling can be viewed through the following lenses:

(1) Improving traditional topic models (e.g., [4, 14]);

(2) Integrating classical topic models with word features [31] or word embeddings [10], or

(3) Embracing the notion (implicit or explicit) of modeling topic embeddings similar to Salakhutdinov and Hinton [33].

In spirit, our goal (see Section 1) is more closely aligned with (3), which forms the basis of review in this section.

Salakhutdinov and Hinton [33] introduce an undirected graphical model, Replicated Softmax, where topics are viewed as hidden binary representations that interact with words in documents. These interactions are specified in the form of weighted connections between topics and words. Motivated by this, Larochelle and Lauly [21] propose the DocNADE (Document Neural Autoregressive Distribution Estimation) model, in which every word is allocated a distinct topic vector as its contextual representation.

The Topical Word Embeddings model is formulated by Liu et al. [24]. Three variants of the Topical Word Embeddings model are proposed where different types of interactions are exploited between topics and words to learn individual topic embeddings. A topic embedding and a word embedding are concatenated to form a topical word embedding for a word. Finally, topical word representations of words in a document are averaged to produce the document representation. Following the same trend, Li et al. [22] develop TopicVec in which topic embeddings are incorporated into the PSDVec model [23]. A link function models the distribution of words for a topic in TopicVec. Considering utilization of learned topic embeddings, He et al. [15] capture topic correlations by considering closeness among topic vectors.

The Neural Variational Document Model [25] and LDA with Product of Experts (ProdLDA) [34] are both Variational AutoEncoder (VAE)-based document models that implicitly capture the topic embeddings in a learnable parameter matrix. Recently, the Embedded Topic Model (ETM) [9], again based on variational inference, incorporates explicit topic embeddings. Due to their representativeness, we consider ProdLDA and ETM as our baselines in this article.

In the following sections, we review several intellectual cornerstones of our work in some detail. 


\subsection{Latent Dirichlet Allocation}

LDA [5] is designed to reveal a global set of latent topics from a large collection of documents, where each latent topic representing a particular theme is a distribution over terms. Each document is viewed as a mixture of topics (in this sense, LDA is similar to soft clustering algorithms). Assuming there are $K$ topics, the generative process of LDA can be formally summarized as follows.

For each document $\mathbf{w}$ in the corpus,

(1) Draw topic proportion $\theta_{\mathbf{w}} \sim \operatorname{Dirichlet}(\alpha)$,

(2) For each word $w_{n}$ in $\mathbf{w}$,

(a) Draw topic assignment $z_{\mathrm{w} n} \sim \operatorname{Mult}\left(\theta_{\mathrm{w}}\right)$,

(b) Draw word $w_{n} \sim \operatorname{Mult}\left(\beta_{z_{\mathrm{w} n}}\right)$,

where $\alpha$ and $\beta$ are the hyper-parameters to control the mixture of topics and the distribution of words per topic, respectively.

Although LDA entertains a distributed topic representation consistent with its BOW view of documents, there are no explicit topic embeddings as in modern deep learning.

The complexity of vanilla LDA amounts to $O\left(N_{\mathrm{w}} K V\right),{ }^{1}$ where $V$ represents the vocabulary size and $N_{\mathrm{w}}$ represents number of tokens in document $\mathbf{w}$. In contrast, the collapsed Gibbs sampling (CGS) version of LDA [13] has a per-document complexity of $O(N K)$, where $N$ represents number of unique tokens in document $\mathbf{w}$. Hence, we use the more efficient CGS-LDA as our baseline in this study.

\subsection{Variational AutoEncoder}

VAE [20] is introduced as a probabilistic version of the standard autoencoder (AE) model. VAE attempts to learn a compressed latent representation vector, $z_{e}(\mathbf{x})$, of the input $\mathbf{x}$ by reconstructing the input as $\mathbf{x}^{\prime \prime}$. The following loss function is the key in the VAE model, which guides the learning process:

$$
\begin{aligned}
L_{v a e} & =E_{q\left(z_{e}(\mathbf{x}) \mid \mathbf{x}\right)}\left[\log \left(p\left(\mathbf{x}^{\prime \prime} \mid z_{e}(\mathbf{x})\right)\right)\right] \\
& -D_{K L}\left(q\left(z_{e}(\mathbf{x}) \mid \mathbf{x}\right) \| p\left(z_{e}(\mathbf{x})\right)\right),
\end{aligned}
$$

where

- $E_{q\left(z_{e}(\mathbf{x}) \mid \mathbf{x}\right)}\left[\log \left(p\left(\mathbf{x}^{\prime \prime} \mid z_{e}(\mathbf{x})\right)\right)\right]$ represents the reconstruction loss,

- $p\left(z_{e}(\mathbf{x})\right)$ is a unit Gaussian $\left(\mu=0\right.$ and $\left.\sigma^{2}=1\right)$ prior over latent embedding vector $z_{e}(\mathbf{x})$, and

- $D_{K L}\left(q\left(z_{e}(\mathbf{x}) \mid \mathbf{x}\right) \| p\left(z_{e}(\mathbf{x})\right)\right)$ represents the KL divergence that forces the posterior, $q\left(z_{e}(\mathbf{x}) \mid \mathbf{x}\right)$ toward the prior, $p\left(z_{e}(\mathbf{x})\right) .^{2}$

Apart from compression, VAE enables probabilistic analysis of latent space that can reveal important hidden structures in the inputs and hence allow to extract more meaningful representation of the data. Moreover, VAE projects the encoded inputs into soft spherical regions [6] in the latent space rather than discrete points. Although VAE enjoys these benefits, the model's performance may deteriorate due to "posterior collapse." According to Bowman et al. [6], a model that encodes important information in latent representation, $z_{e}(\mathbf{x})$, will have a low reconstruction loss and a non-zero KL divergence. However, training VAE-based models becomes a challenge due to vanishing KL divergence; specifically, variational posterior distribution $q\left(z_{e}(\mathbf{x}) \mid \mathbf{x}\right)$ is consistently set

\footnotetext{
${ }^{1}$ https://stats.stackexchange.com/questions/331827/latent-dirichlet-allocation-complexity-and-implementation-details.

${ }^{2}$ Unlike AE, $q\left(z_{e}(\mathbf{x}) \mid \mathbf{x}\right)$ represents a probability distribution in VAE.
} 


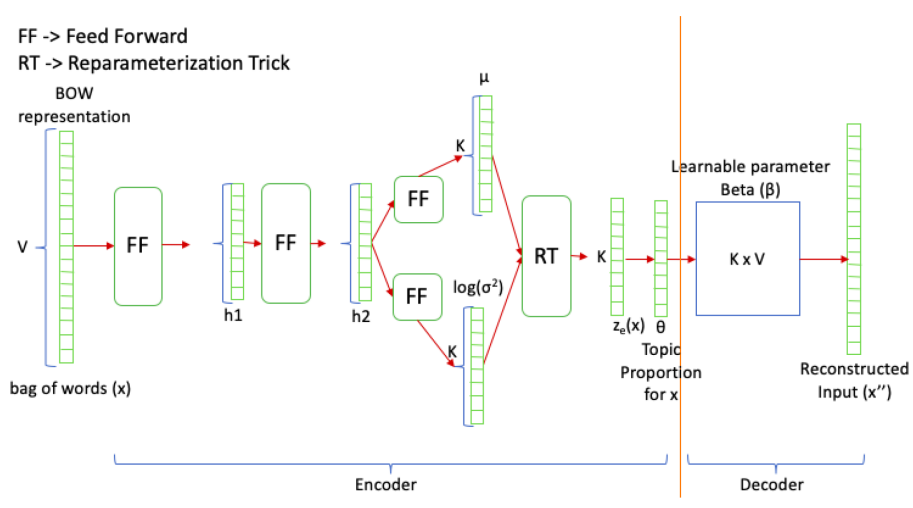

Fig. 1. ProdLDA.

equal to prior $p\left(z_{e}(\mathbf{x})\right)$. At this point, latent information in $z_{e}(\mathbf{x})$ becomes irrelevant while reconstructing the input as $\mathbf{x}^{\prime \prime}$. To overcome the posterior collapse issue, many innovative approaches have been proposed, including adjusting optimization parameters (e.g., learning rate, type of optimizer), diminishing the effect of KL divergence by weighing it down in Equation (1), or utilizing techniques such as batch normalization and dropout.

\subsection{VAE-Based Topic Models}

Leveraging the VAE architecture, Srivastava and Sutton [34] propose the ProdLDA model to compute $K$ topics from the corpus. The authors argue that VAE can map a document to a well-behaved approximate posterior distribution $\left(q\left(z_{e}(\mathbf{x}) \mid \mathbf{x}\right)\right)$ by using an inference neural network. Moreover, this neural network is expected to perform probabilistic inference that is at the core of LDA-family models. As shown in Figure 1, ProdLDA starts with a BOW representation of a document, x. Next, several non-linear transformations are applied to produce the hidden representation h2 of $\mathbf{x}$, followed by linear transformations applied on h2 to compute a $K$-dimension mean vector, $\mu$, and a log-variance vector, $\sigma^{2}$, of the same dimension. A generative network takes over from here. The reparameterization trick [20] is applied to sample a unit Gaussian vector, eps, of dimension $K$. At this point, the sampled latent representation, $z_{e}(\mathbf{x})$, is formulated by using Equation (2). A softmax function is applied on $z_{e}(\mathbf{x})$ to retrieve topic proportions, $\theta_{k}$ (Equation (3)), which results in a vector $\theta$ for input $\mathbf{x}$. Then, a reconstructed input, $\mathbf{x}^{\prime \prime}$, is fetched by utilizing a learned parameter, $\beta_{\text {prodlda }}$ (Equation (4)). Finally, the loss function in Equation (1) is employed to train the whole network.

$$
\begin{gathered}
\left.z_{e}(\mathbf{x})=\mu+\sqrt{\exp \left(\log \sigma^{2}\right.}\right) * \text { eps } \\
\theta_{k}=\frac{\exp \left(z_{e}(\mathbf{x})_{j}\right)}{\sum_{k=0}^{K-1} \exp \left(z_{e}(\mathbf{x})\right)} \\
\mathbf{x}^{\prime \prime}=\theta \times \beta_{\text {prodlda }}
\end{gathered}
$$

In this model, $\beta_{\text {prodlda }}$ captures topic-word relationships. Specifically, the $k$-th row represents the $k$-th topic, which is a distribution over the vocabulary. This is essentially the same as LDA due to the BOW input. To tackle posterior collapse, ProdLDA tweaks the optimization parameters and utilizes batch normalization with dropout technique. Per-document complexity of the ProdLDA model is $O(V h+V K)$, where $h$ represents dimension of hidden representations, such as h2, and $V$ represents vocabulary size. 


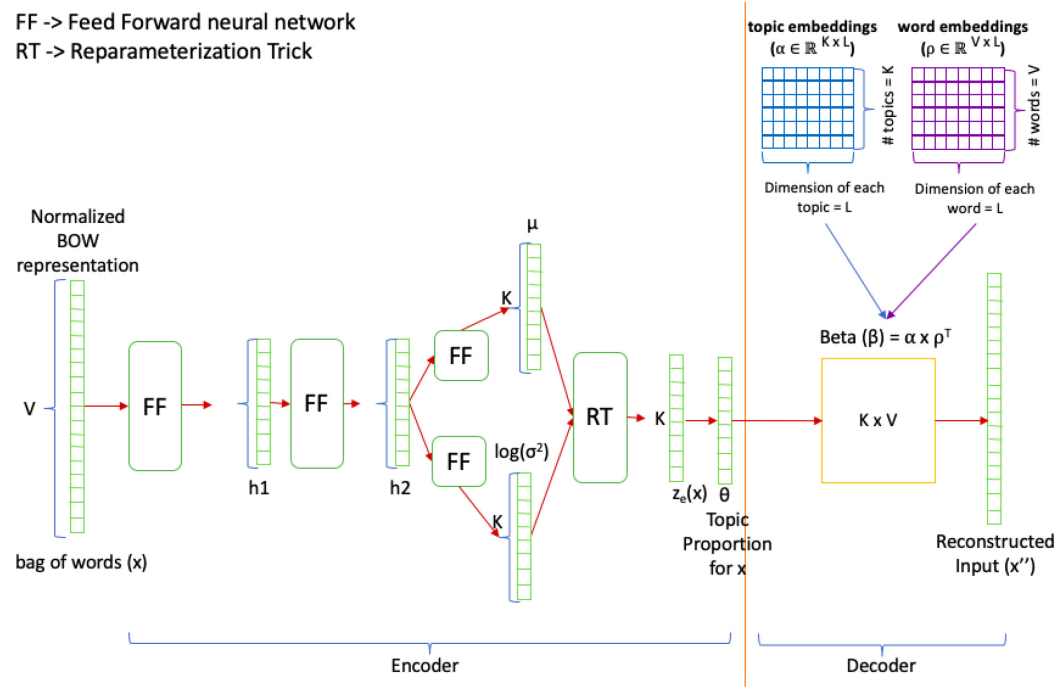

Fig. 2. Embedded Topic Model.

Although ProdLDA is a neural model built on the strength of VAE, it consumes BOW-style input documents and produces BOW-style topics, due to its focus on mimicking LDA behavior. It lacks the following:

- Explicit dense topic embeddings as model output,

- Natural input document representations by utilizing distributed representations of word semantics (i.e., word embeddings).

ETM [9], as shown in Figure 2, attempts to overcome some of ProdLDA's weaknesses. Specifically, the authors incorporate topic embeddings, $\alpha \in \mathbb{R}^{\mathrm{K} x \mathrm{~L}}$, and utilize word embeddings, $\rho \in$ $\mathbb{R}^{\mathrm{VxL}}$, to formulate $\beta_{\text {etm }} \in \mathbb{R}^{\mathrm{K} \times \mathrm{V}}$. Formally,

$$
\beta_{\text {etm }}=\alpha \times \rho^{T} .
$$

According to Dieng et al. [9], $\alpha \in \mathbb{R}^{\mathrm{K} \times \mathrm{L}}$ learns the topic representations during the training process that allows for better topic interpretability (TI). Every topic is now a $L$-dimension vector, $\alpha_{k}$, in ETM rather than a $V$-dimension vector, $\beta_{k}$, in ProdLDA. In contrast to ProdLDA, ETM leverages dropout without batch normalization, however, with decent optimization parameters to avoid posterior collapse. Per-document complexity of ETM is $O(V h+V L K)$.

While achieving the goal of learning explicit topic embeddings, surprisingly enough, ETM still utilizes a BOW-style input document representation. Due to the continuous nature of the latent vector $z_{e}$, the somewhat unnatural interaction between the word-embedding matrix $\rho$ and topicembedding matrix $\alpha$ occurs late in the system pipeline. We believe that these shortcomings present opportunities for improvement, and vector quantization seems to be a powerful vehicle.

\subsection{Vector Quantized-Variational AutoEncoder}

The vector quantization (VQ) technique introduced by Gray [12] is originally used to compress data and signals sent over a communication channel (see Appendix A). The technique has been shown to result in 
- Production of latent and accurate descriptions of input signals,

- Transmission of high-quality images and speeches over a finite capacity of channel, and

- Reduction in storage capacity of a medium.

Vector Quantized-Variational AutoEncoder (VQ-VAE) [37] integrates vector quantization with the VAE framework by learning discrete latent representations in a generative reconstruction setting. Overall, the VQ-VAE model can be summarized as follows:

(1) Let us assume that there exists a latent embedding space, $\mathbf{E} \in \mathbb{R}^{\mathrm{Kxd}}$, where $K$ and $d$ represent the number of latent vectors and the dimension of each latent vector, respectively.

(2) A raw input $\mathbf{x}$ is provided to the encoder that outputs a discrete encoded representation, $z_{e}(\mathbf{x}) \in \mathbb{R}^{\mathrm{d}}$.

(3) Next, $z_{e}(\mathbf{x})$ is transmitted through a discretization bottleneck using a nearest-neighbor (Euclidean distance) lookup on embedding space, E. Formally,

$$
z_{q}(\mathbf{x})=E_{k}, k=\arg \min _{t_{i} \in K}\left\|z_{e}(\mathbf{x})-E_{t_{i}}\right\|_{2},
$$

where

- $z_{q}(\mathbf{x}) \in \mathbb{R}^{\mathrm{d}}$ represents the closest latent vector (quantized representation) retrieved from space $\mathbf{E}$ and is the input to the decoder;

- $t_{i}$ represents the index of a latent vector, $E_{t_{i}}$, in embedding space $\mathbf{E}$; and

- $k$ represents the index of the closest latent embedding vector based on $l_{2}$ distance.

(4) Finally, $z_{q}(\mathbf{x})$ is sent to a decoder that outputs a reconstructed input, $\mathbf{x}^{\prime \prime}$.

(5) During the process, the following loss function is minimized:

$$
\begin{aligned}
L_{v q-v a e}= & l_{\text {reconstruct }}+l_{v q} \\
l_{v q}= & \left\|s g\left(z_{e}(\mathbf{x})\right)-z_{q}(\mathbf{x})\right\|_{2}^{2} \\
& +\gamma\left\|z_{e}(\mathbf{x})-s g\left(z_{q}(\mathbf{x})\right)\right\|_{2}^{2},
\end{aligned}
$$

where

- $l_{\text {reconstruct }}$ represents the reconstruction loss;

- $l_{v q}$ represents the VQ loss;

- the first term of $l_{v q}$ ensures that embeddings in latent space are updated during training;

- the second term (referred to as commitment loss) of $l_{v q}$ ensures that the encoder commits to an embedding in latent space;

- $s g$ represents the stopgradient operator that is an identity during forward pass and has zero partial derivatives; and

- $\gamma$ is a hyper-parameter to control the third term, commitment loss, in the total loss.

By using discrete latent representations, VQ-VAE is less susceptible to posterior collapse. According to Oord et al. [37], VQ-VAE considers a deterministic variational posterior distribution, $q\left(z_{e}(\mathbf{x}) \mid \mathbf{x}\right)$ (one-hot), and a uniform prior, $p\left(z_{e}(\mathbf{x})\right)$. This results in constant KL divergence equal to $\log (K)$ that can be ignored during the training process and makes VQ-VAE insensitive to KL divergence.

VQ-VAE is proposed in the context of continuous-valued input, and the introduction of VQ is largely motivated by data compression. As a result, the model is applied quite naturally on image, audio, and video data. We believe that VQ-based thinking is general enough to work with discretevalued input such as text, since VQ is a general form of clustering-like pattern recognition where an input pattern is matched against a stored set of latent codes [11]. In this work, with topic modeling viewed as soft clustering of words, the latent codes nicely correspond to the notion of latent topics. 


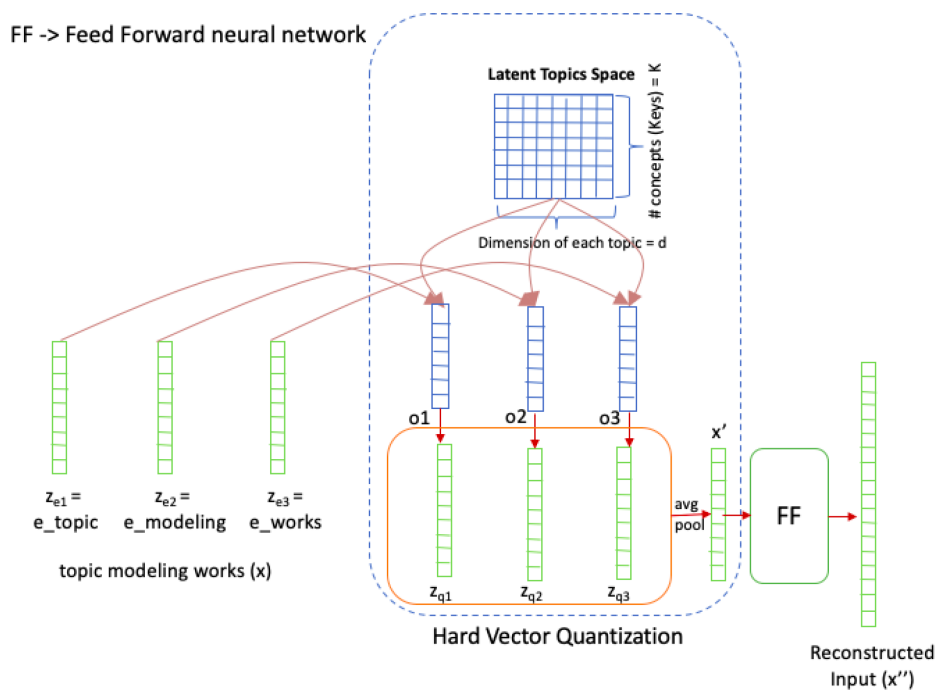

Fig. 3. Hard VQ-TM.

Such conceptualization allows much more subtle interaction between word embeddings and topic embeddings, which is absent in ProdLDA and weak in ETM.

\section{VECTOR-QUANTIZATION-BASED TOPIC MODELS}

Motivated by the pursuit of explicit and dense topic embeddings, and taking advantage of lessons learned from ProdLDA and ETM, we propose several versions of vector-quantization-based topic models (VQ-TMs). In the spirit of bona fide distributed representations in modern deep learning, all models consume word embeddings (dense vectors) instead of BOW as input.

\subsection{Hard VQ-TM}

This model is similar in spirit to VQ-VAE working with word embeddings as input, with our own novel loss term.

As shown in Figure 3, we view the latent embedding space, $\mathbf{E} \in \mathbb{R}^{\mathrm{K} x \mathrm{~d}}$ (see step 1 in VQ-VAE), as a latent topic space (LTS) that contains a set of latent topics where $K$ represents number of distinct topics. Similar to $z_{e}(\mathbf{x})$ (see step 2 in VQ-VAE), a discrete encoded representation $z_{e n} \in \mathbb{R}^{\mathrm{d}}$ for every word is considered where $n$ is the index of words in the document $\mathbf{x}$. In this work, $z_{e n}$ is equal to word-embedding $e_{n} \in \mathbb{R}^{\mathrm{d}}$ for word $w_{n}$, as we do not use any structural encoder. $z_{\text {en }}$ then also behaves as a query that interacts (nearest-neighbor lookup) with LTS to retrieve the closest index, $k$, and correspondingly the closest latent vector, $z_{q n}$ (see step 3 in VQ-VAE). At this point, we interpret the retrieved index, $k$, as producing an implicit value vector, $o_{n}$, a one-hot vector. Different from step 4 in VQ-VAE, a transformed document representation, $\mathbf{x}^{\prime}$, is computed by applying the average pooling on all of the retrieved latent vectors, $z_{q n} \mathrm{~s}$, as shown in Equation (8). As an output of this step, $\mathbf{x}^{\prime}$ can be considered as a mixture of concepts closest to all tokens in the document:

$$
\mathbf{x}^{\prime}=\frac{\sum_{1}^{n} z_{q n}}{n},
$$

where

- $z_{q n} \in \mathbb{R}^{\mathrm{d}}$ represents the closest latent vector (quantized representation) from LTS, E, for word $w_{n}$, and

- $n$ is the total number of words in the document, $\mathbf{w}$.

ACM Transactions on Intelligent Systems and Technology, Vol. 12, No. 3, Article 34. Publication date: April 2021. 
Ultimately, $\mathbf{x}^{\prime}$ is transmitted through a feed forward (FF) neural network to produce a reconstructed input, $\mathbf{x}^{\prime \prime}$.

$$
\mathbf{x}^{\prime \prime}=\mathrm{FF}\left(\mathbf{x}^{\prime}\right)
$$

Since topic embeddings are learned during the training process, we conjecture that they may come close to each other in the latent space due to movement induced by the first term in $l_{v q}$ of Equation (7). In principle, however, topic embeddings should be as distinct as possible for better topic interpretability. Therefore, we augment $L_{v q-v a e}$ in Equation (7) by adding a Latent Topics Space $\left(l_{l t s}\right)$ loss. Formally,

where

$$
l_{l t s}= \begin{cases}d_{t_{i} t_{j}}, & t_{i}==t_{j} \\ \max \left\{0, \Delta-d_{t_{i} t_{j}}\right\} & t_{i} \neq t_{j}\end{cases}
$$

- $i \in\{0, \mathrm{~K}-1\}$ and $j \in\{0, \mathrm{~K}-1\}$;

- $t_{i}$ and $t_{j}$ represent the indexes of topic embeddings $E_{t_{i}}$ and $E_{t_{j}}$, respectively, in LTS E;

- $d_{t_{i} t_{j}}$ is the $l_{2}$ distance between topics $E_{t_{i}}$ and $E_{t_{j}}$;

- $\Delta$ represents a hyper-parameter that is set to 1 .

Intuitively, $l_{l t s}$ is a form of Hinge Embedding Loss that pushes for dis-similarity between concepts in LTS. Now the total loss function becomes

$$
L_{v q-t m}=l_{\text {reconstruct }}+l_{v q}+\zeta l_{l t s}
$$

where $\zeta$ is a hyper-parameter and is set to 0.001 . We term this model Hard VQ-TM because for each word it only extracts a single (hard assignment) latent topic from the LTS. Per-document complexity of Hard VQ-TM is given by $O\left(N_{\mathrm{w}} K+V d\right)$, where $N_{\mathrm{w}}$ represents the number of tokens in document $\mathbf{w}$.

As our first VQ-based topic model, Hard VQ-TM has the following benefits:

- By modeling LTS, it computes explicit topic embeddings.

- It represents input documents by utilizing word embeddings.

However, by assigning only one topic embedding to each word, Hard VQ-TM misses an opportunity to directly model the subtle interaction between words and topics. It leads to our next model, Soft VQ-TM.

\subsection{Soft VQ-TM}

As mentioned in Section 3.1, Hard VQ-TM utilizes a one-hot value vector, $o_{n}$, to commit a word $w_{n}$ to an individual concept in the document. Intuitively, it can be interpreted as a word expressing one specific meaning in the whole document. However, such "hard" alignment between words and topics may not suffice in preserving the semantic richness of natural language. Therefore, our second model, Soft VQ-TM, makes an attempt to "softly" associate multiple concepts with each word $w_{n}$. This notion is absent not only in Hard VQ-TM but also in ProdLDA and ETM. In Soft VQ$\mathrm{TM}$, the one-hot value vector, $o_{n}$, is substituted by an attention vector, $a_{n}$ (Figure 4 ), which contains normalized attention scores, $a_{n t_{i}} \mathrm{~s}$, where $i \in\{0, \mathrm{~K}-1\}$. Effectively, $a_{n t_{i}}$ s represent soft associations between words in the document and various topics available in E. Formally, attention scores are computed by Equations (12) and (13):

$$
\begin{gathered}
u_{n t_{i}}=z_{e n} \times E_{t_{i}}^{T}, \\
a_{n t_{i}}=\frac{\exp \left(u_{n t_{i}}\right)}{\sum_{i=0}^{K-1} \exp \left(u_{n t_{i}}\right)},
\end{gathered}
$$




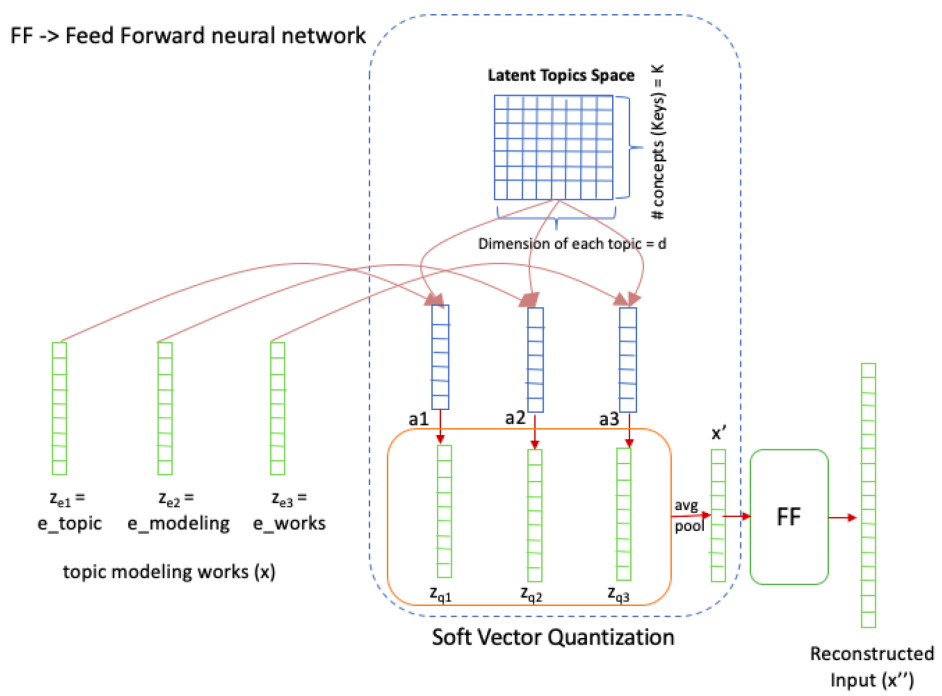

Fig. 4. Soft VQ-TM.

where

- $\times$ represents matrix multiplication;

- $n$ represents the index of word in the document;

- $t_{i}$ represents the index of topic, $E_{t_{i}} \in \mathbb{R}^{\mathrm{d}}$, in LTS E;

- $z_{e n} \in \mathbb{R}^{\mathrm{d}}$ represents the discrete encoded representation of word $w_{n}$ in the document. In this work, $z_{e n}$ is equal to word-embedding $e_{n}$ for word $w_{n}$;

- $u_{n t_{i}}$ represents the un-normalized score between word $w_{n}$ and topic $E_{t_{i}}$ from LTS E; and

- $a_{n t_{i}}$ represents the normalized attention score between word $w_{n}$ and topic $E_{t_{i}}$ from LTS E.

Next, a weighted quantized representation, $z_{q n}$, is formulated for word $w_{n}$ as shown in Equation (14).

$$
z_{q n}=\sum_{i=0}^{K-1} a_{n t_{i}} * E_{t_{i}},
$$

where

- $z_{q n} \in \mathbb{R}^{\mathrm{d}}$ represents the weighted quantized representation for word $w_{n}$ and

- $E_{t_{i}} \in \mathbb{R}^{\mathrm{d}}$ represents the topic $t_{i}$ from LTS $\mathbf{E}$.

Intuitively, Soft VQ-TM transforms a word $w_{n}$ s' un-modified embedding, $z_{e n}=e_{n}$, into a modified embedding $z_{q n}$ that contains polysemous information about word $w_{n}$. Next, to retrieve $\mathbf{x}^{\prime}$ (Equation (8)) and $\mathbf{x}^{\prime \prime}$ (Equation (9)), we follow the same procedure as mentioned in Section 3.1. Finally, we employ the loss function in Equation (11). Per-document complexity of Soft VQ-TM is $O\left(N_{\mathrm{w}} d K+V d\right)$.

In summary, Soft VQ-TM builds upon Hard VQ-TM but strengthens it by softly associating multiple topics with a given word. This is a major conceptual leap where each word is now a mixture of topics, whereas in the LDA family (including ProdLDA and ETM), only documents are viewed as mixtures of topics. 


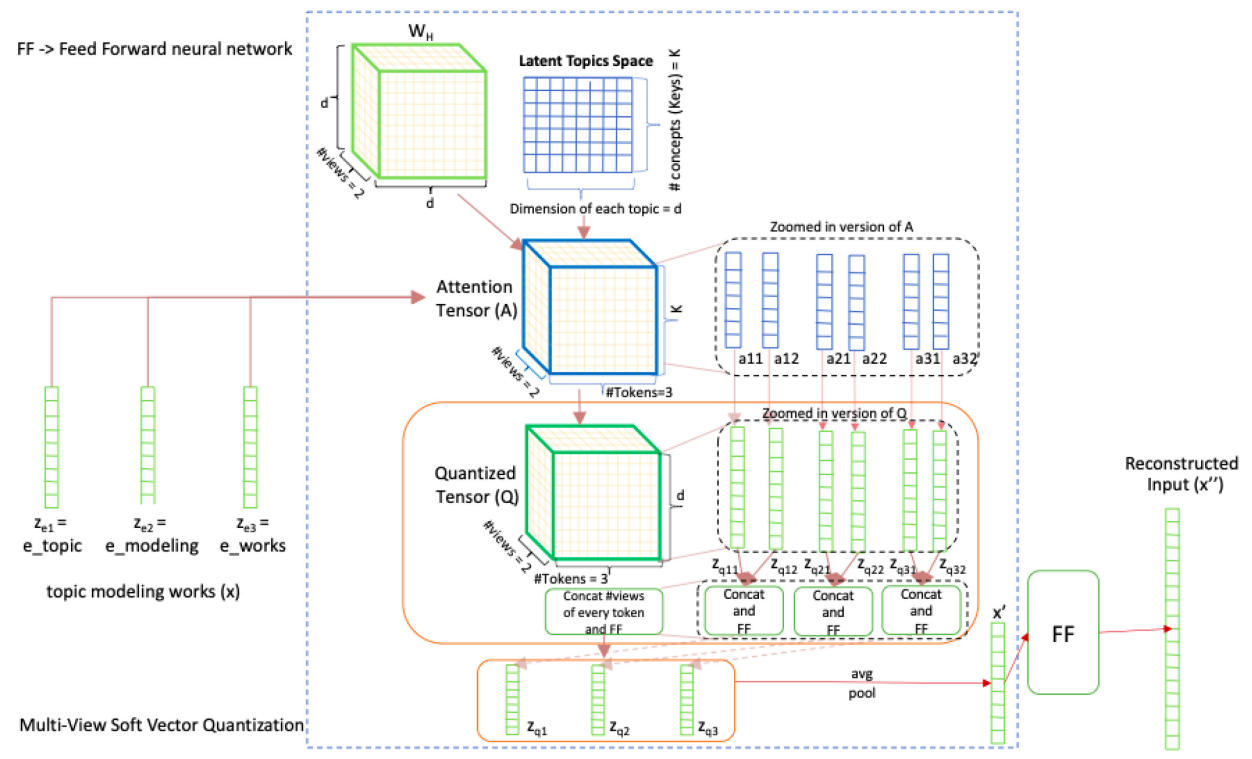

Fig. 5. Multi-View Soft VQ-TM $\left(H=2, \mathbf{W}_{H}\right.$ contains multiple views).

\subsection{Multi-View Soft VQ-TM}

To further push the envelope, and inspired by previous work (e.g., $[8,38])$, we propose the MultiView Soft VQ-TM model to enable multiple soft associations between words and topics by using multiple views $\left(W_{H} \in \mathbb{R}^{\mathrm{Hxdxd}}\right.$ in Figure 5). These non-identical views effectively implement a multi-head attention mechanism, and are expected to capture semantic richness and reveal subtle structures. Specifically, they produce an attention tensor $\left(A \in \mathbb{R}^{\mathrm{H} \times \mathrm{Nwx} K}\right)$ containing attention vectors $\left(a_{n h} \mathrm{~s}\right)$ related to all views $(H)$ for all words $N_{\mathbf{w}}$ in document w. For example, in Figure 5, the number of views is set to 2 , which results in two attention vectors (zoomed-in version of $A$ ) for every word. Formally, Equation (15) is utilized to compute the attention tensor $(A)$ :

$$
\begin{aligned}
U & =E_{\mathrm{w}} \times\left(W_{H} \times \mathbf{E}^{T}\right) \\
A & =\operatorname{softmax}(U),
\end{aligned}
$$

where

- $\times$ represents matrix multiplication,

- $E_{\mathrm{w}}$ represents the embedding matrix of all words $N_{\mathrm{w}}$ in document $\mathbf{w}$,

- $W_{H} \in \mathbb{R}^{\mathrm{Hxdxd}}$ is a learned matrix containing $H$ views,

- $U \in \mathbb{R}^{\mathrm{H} \times \mathrm{Nw} \times \mathrm{K}}$ represents un-normalized scores between $E_{\mathrm{w}}$ and LTS E using $H$ views,

- $\operatorname{softmax}(U)$ represents normalization of un-normalized scores along all topics $K$, and

- $A \in \mathbb{R}^{\mathrm{H} \times \mathrm{Nw} \times \mathrm{K}}$ represents the attention tensor.

Next, a weighted quantized tensor $\left(Q \in \mathbb{R}^{\mathrm{HxNwxd}}\right)$ is formulated (Equation (16)) that contains modified embeddings $z_{q n h}$ s (see zoomed-in version of $Q$ in Figure 5) related to all views for all of the words in document $\mathbf{w}$ :

$$
Q=A \times \mathbf{E},
$$

where

- $\times$ represents matrix multiplication. 
Then, we open the quantized-tensor Q (Equation (17)) as below to fetch word and view-specific $z_{q n h}$ :

$$
z_{q n h}=\operatorname{unbind}(Q),
$$

where

- $\operatorname{unbind}(Q)$ represents a slice-operation to retrieve word and view specific quantized representation $z_{q n h}$.

Multi-View Soft VQ-TM transforms word $w_{n}$ s' un-modified embedding, $z_{e n}=e_{n}$, to a viewspecific modified embedding $z_{q n h}$. Finally, all $z_{q n h} \mathrm{~s}$ for a word $w_{n}$ are blended together by utilizing Equation (18) to form a single quantized representation, $z_{q n}$ :

$$
z_{q n}=\tanh \left(\mathrm{FF}\left(\operatorname{concat}_{i=0}^{H-1}\left(z_{q n h_{i}}\right)\right)\right),
$$

where

- $z_{q n} \in \mathbb{R}^{\mathrm{d}}$ represents the concatenated quantized representation for word $w_{n}$,

- FF represents the feed-forward neural network, and

- concat represents the concatenated operation.

Again, to compute $\mathbf{x}^{\prime}$ (Equation (8)) and $\mathbf{x}^{\prime \prime}$ (Equation (9)), we follow the same procedure as mentioned in Section 3.1. We employ the loss function in Equation (11). Per-document complexity of the Multi-View Soft VQ-TM model is $O\left(N_{\mathrm{w}} H d^{2} K+V d\right)$.

Note: In all three proposed models, we purposefully refrain from using any structural encoder/decoder based on contextual information (e.g., LSTM [17]), although our architectures easily allow such integration. The two main reasons are

- To demonstrate the effectiveness of VQ by limiting the capacity of encoder/decoder, and

- To facilitate fair comparison with all LDA-inspired algorithms (including ProdLDA and ETM) that do not use any strong encoder/decoder.

\section{EXPERIMENTAL SETUP}

To perform systematic evaluation, we compare our VQ-TMs with three representative baselines: CGS-LDA, ProdLDA, and ETM. Unlike CGS-LDA and ProdLDA, whose topic representations are directly based on word distributions, ETM and VQ-TMs explicitly learn dense topic embeddings. As a result, the topic-word association matrix, a critical data structure for Topic Interpretability (TI), is instantiated in different forms in all of the models. It is innate in CGS-LDA, whereas it is a learned $\beta_{\text {prodlda }}$ matrix in ProdLDA; in ETM and VQ-TMs, the matrix (notated as $\beta_{\text {etm }}, \beta_{\text {hard-vqtm }}$, $\beta_{\text {soft-vqtm }}$, and $\beta_{\text {multi-view-soft-vqtm }}$ ) is computed as the product of the word-embedding matrix and the topic-embedding matrix.

\subsection{Evaluation Metrics}

We consider the following evaluation metrics:

(1) Perplexity (PPL), originated from the document modeling task, has been widely used for evaluating topic models since the work of Blei et al. [5] and is arguably the most important performance measure. It is equivalent to the inverse of the geometric mean per-word likelihood. Lower PPL indicates a better generative model. Formally, assuming that there are $\mathbf{M}$ documents in test dataset $D_{\text {test }}$, we use the following equation to calculate 
per-word PPL:

$$
\operatorname{ppl}\left(D_{\text {test }}\right)=\exp \left(\frac{-\sum_{j=1}^{M} \ln \left(p\left(\mathbf{w}_{j}\right)\right)}{\sum_{j=1}^{M} N_{j}}\right),
$$

where

(a) $p(\mathbf{w})$ represents the probability of document $\mathbf{w}$, and

(b) $N_{j}$ represents the total number of tokens in the document $\mathbf{w}$.

(2) Topic Coherence (TC), proposed by Newman et al. [27], is a prevalent measure to illustrate the quality of topics. It quantifies the consistency of a topic by computing the mutual information among $C$ (10 in this work) representative words for that topic. Higher average TC represents better topic quality. Srivastava and Sutton [34] and Dieng et al. [9] utilize normalized pointwise mutual information to indicate a meaningful topic. However, Röder et al. [32] conduct a systematic study for various coherence measures and concludes that $C_{V}$, a combination of indirect cosine measure, normalized pointwise mutual information, and Boolean sliding window, is better than other relevant measures. Authors have shared their work as a tool, Palmetto, ${ }^{3}$ which we utilize to calculate the $C_{V}$ metric per topic, and report the average over all the topics $(A T C)$.

(3) Topic Diversity (TD) ${ }^{4}$ [9] quantifies redundancy across topics. Diversity near 1 indicates low redundancy, and a value close to 0 implies repetitive topics. Formally, it is calculated as

where

$$
T D=\frac{n\left(W_{\text {unique }}\right)}{C * K},
$$

- $C$ is the number of top representative words for any topic;

- $K$ is the number of topics; and

- $n\left(W_{\text {unique }}\right)$ represents the number of unique words available in set $W_{\text {unique }}=\bigcup_{i=0}^{K-1} W_{i}$, where $W_{i}$ is the set of top $C$ representative words for the $i$-th topic.

(4) Topic Interpretability (TI), again defined by Dieng et al. [9], is dependent on TC and TD. Hence, in this work, we calculate TD as shown in Equation (21).

$$
T I=A T C * T D
$$

\subsection{Datasets}

We use the following datasets for our experiments:

(1) 20 Newsgroups (20 Ng) [19] is a widely used dataset in topic modeling and information retrieval. For fair comparison, we utilize the pre-processed dataset made available by Srivastava and Sutton [34]. Specifically, the following pre-processing steps are performed: tokenization and removal of non UTF-8 characters and English stop words from the dataset, which result in a vocabulary size of 2,000. Training corpus consists of 11,000 instances. We take 5\% (375) of the original test instances as the validation dataset and use the rest $(7,000)$ of the instances as the test corpus.

(2) Conala Corpus ${ }^{5}$ contains 2,379 and 500 English-Python pairs in the training and test set, which are manually curated. Additionally, there are 598,237 pairs that have been mined using the system developed by Yin et al. [40]. In this work, we use 42,500, 2,500, and 5,000 NL

\footnotetext{
${ }^{3}$ Available at https://github.com/dice-group/Palmetto.

${ }^{4}$ Both TD and TI are newly proposed evaluation metrics. Their validity is yet to be more systematically studied, which is outside the scope of our research.

${ }^{5}$ Available at https://conala-corpus.github.io/.
} 
(English) sentences as the training, validation, and test set for topic modeling. However, we use the same dataset (plus corresponding Python snippets) for the code generation task (see Section 6). Again, we perform the same pre-processing steps as described for 20 $\mathrm{Ng}$, which result in an English vocabulary size of 8,000 for this dataset. Last, for Python code snippets, tokenization is performed, which yields 40,000 code tokens.

\subsection{Model Training}

First, we utilize the mallet ${ }^{6}$ tool to conduct experiments for the CGS-LDA model. We vary number of topics, $K \in\{20,50,100\}$, and use default hyper-parameters during the training step. Next, for ProdLDA and ETM, we keep the same configuration as mentioned by Srivastava and Sutton [34] and Dieng et al. [9], respectively. Specifically, the hidden representation, h2, in ProdLDA has a dimension of 100, whereas it is 800 in ETM. For ProdLDA, the batch size is set to 200, the learning rate is kept at 0.002 , and the number of epochs is equal to 100 . However, these hyper-parameters are set to 1,000, 0.005, and 150, respectively, for ETM as mentioned by Dieng et al. [9]. Additionally, 300-dimensional topic embeddings and GloVE word embeddings [30] are utilized in ETM.

For fair comparison, we also employ 300-dimensional (d) embedding space and GloVE word embeddings in all variants of proposed VQ-TMs. Word embeddings are kept fixed during the training procedure, irrespective of the model implemented. Furthermore, we use a learning rate of 0.0002 and a batch size of 50. Specific to Multi-View Soft VQ-TM, we experiment with two and eight views.

A commitment cost ( $\gamma$ in $l_{v q}$ in Equation (11)) of 0.25 is implemented for all VQ-TMs. Finally, the Adam optimizer is applied for all neural models.

\section{RESULTS AND DISCUSSION}

\subsection{Experimental Results with Qualitative Analysis}

Table 1 portrays experimental results for the $20 \mathrm{Ng}$ and Conala test sets. In Tables 2 and 3, we also provide the top-word lists per topic learned by various models from the $20 \mathrm{Ng}$ corpus.

Overall, the VQ-TM family exhibits dominant strength across both datasets and all evaluation metrics, with the exception of ETM leading on TD for the Conala dataset. In the following sections, we inspect the results and present a qualitative analysis.

5.1.1 Perplexity. Considering the PPL results in Table 1, the VQ-TM models consistently outperform baselines. To better understand this behavior, we visualize two revealing data structures: (1) Document-Topic mixture vector ( $\theta$ 's in Figure 6) and (2) Topic-Word association matrices ( $\beta$ 's in Figure 7), produced by all models. It must be noted that the figures are specific to dataset $20 \mathrm{Ng}$ and should be read in conjunction with Tables 2 and 3, which show the topics learned by CGSLDA, ETM, Hard VQ-TM, Soft VQ-TM, and Multi-View Soft VQ-TM when the number of topics $K=20$.

In Figure 6, we observe that the VQ-TM model family tends to produce "softer" per-document topic mixtures. For example, the Multi-View Soft VQ-TM model is able to pick subtle and relevant topics (Topics 1 and 4) in addition to the obvious topic: game (see Tables 2 and 3; captured as Topic 13 in CGS-LDA, Topic 9 in ETM, Topic 14 in Hard VQ-TM, Topic 18 in Soft VQ-TM, and Topic 12 in Multi-View Soft VQ-TM (\#Views=8)). Specifically, in Table 3, we can see that for Multi-View Soft VQ-TM (\#Views=8), Topic 1 modestly conveys the notion of named entities and Topic 4 the notion of business and economics. Similar patterns can be observed in other documents. By capturing more

$\overline{{ }^{6} \mathrm{http}: / / \text { mallet.cs.umass.edu/topics.php. }}$ 
Table 1. Results on the $20 \mathrm{Ng}$ and Conala Test Sets: Number of Topics, $K \in\{20,50,100\}$

\begin{tabular}{|c|c|c|c|c|c|c|}
\hline Dataset & Model & $\begin{array}{c}\text { \#Topics } \\
K\end{array}$ & PPL & Avg. TC & TD & TI \\
\hline \multirow{21}{*}{$20 \mathrm{Ng}$} & CGS-LDA & \multirow{7}{*}{20} & 1553.2250 & 0.3868 & 0.7600 & 0.2939 \\
\hline & ProdLDA & & 952.6496 & 0.4500 & 0.7300 & 0.3350 \\
\hline & ETM & & 770.9706 & 0.3822 & 0.8150 & 0.3115 \\
\hline & Hard VQ-TM & & 755.6444 & 0.4500 & 0.9000 & 0.4045 \\
\hline & Soft VQ-TM & & 716.3365 & 0.4531 & 0.9350 & 0.4237 \\
\hline & Multi-View Soft VQ-TM (\#Views=2) & & 598.5253 & 0.4485 & 0.8800 & 0.3947 \\
\hline & Multi-View Soft VQ-TM (\#Views=8) & & 447.7766 & 0.4507 & 0.9150 & 0.4124 \\
\hline & CGS-LDA & \multirow{7}{*}{50} & 1416.3801 & 0.3964 & 0.7140 & 0.2830 \\
\hline & ProdLDA & & 1000.1553 & 0.4021 & 0.6180 & 0.2485 \\
\hline & ETM & & 693.6952 & 0.3853 & 0.7000 & 0.2697 \\
\hline & Hard VQ-TM & & 659.3923 & 0.4443 & 0.8260 & 0.3670 \\
\hline & Soft VQ-TM & & 649.8460 & 0.4499 & $\underline{0.8400}$ & $\underline{0.3779}$ \\
\hline & Multi-View Soft VQ-TM (\#Views=2) & & 579.9109 & $\underline{0.4536}$ & 0.6780 & 0.3075 \\
\hline & Multi-View Soft VQ-TM (\#Views=8) & & 398.5577 & 0.4022 & 0.5360 & 0.2156 \\
\hline & CGS-LDA & \multirow{7}{*}{100} & 1357.0076 & 0.3935 & 0.6020 & 0.2369 \\
\hline & ProdLDA & & 1046.6184 & 0.3953 & 0.6000 & 0.2372 \\
\hline & ETM & & 652.9964 & 0.3861 & 0.5940 & 0.2293 \\
\hline & Hard VQ-TM & & 599.8653 & 0.4321 & 0.7120 & $\underline{0.3077}$ \\
\hline & Soft VQ-TM & & 607.4926 & 0.4270 & 0.7170 & 0.3062 \\
\hline & Multi-View Soft VQ-TM (\#Views=2) & & 562.6996 & 0.4608 & 0.3330 & 0.1534 \\
\hline & Multi-View Soft VQ-TM (\#Views=8) & & 391.7103 & 0.3917 & 0.2610 & 0.1022 \\
\hline \multirow{21}{*}{ Conala } & CGS-LDA & \multirow{7}{*}{20} & 287.4898 & 0.3985 & 0.7650 & 0.3048 \\
\hline & ProdLDA & & 117.0318 & 0.3989 & 0.8500 & 0.3391 \\
\hline & ETM & & 89.4331 & 0.4023 & 1.0000 & 0.4023 \\
\hline & Hard VQ-TM & & 56.4504 & 0.4399 & 0.9200 & 0.4050 \\
\hline & Soft VQ-TM & & 51.6614 & 0.4533 & 0.9350 & 0.4238 \\
\hline & Multi-View Soft VQ-TM (\#Views=2) & & 24.1174 & 0.4772 & 0.9700 & 0.4629 \\
\hline & Multi-View Soft VQ-TM (\#Views=8) & & 12.5639 & $\underline{0.5102}$ & 0.9650 & 0.4923 \\
\hline & CGS-LDA & \multirow{7}{*}{50} & 267.9689 & 0.4013 & 0.5980 & 0.2400 \\
\hline & ProdLDA & & 118.0256 & 0.3902 & 0.8220 & 0.3208 \\
\hline & ETM & & 65.9982 & 0.4031 & 0.9620 & 0.3878 \\
\hline & Hard VQ-TM & & 36.8218 & 0.4463 & 0.8880 & 0.3963 \\
\hline & Soft VQ-TM & & 35.6052 & 0.4599 & 0.9020 & 0.4148 \\
\hline & Multi-View Soft VQ-TM (\#Views=2) & & 15.0803 & $\underline{0.4842}$ & 0.9020 & 0.4368 \\
\hline & Multi-View Soft VQ-TM (\#Views=8) & & 11.6983 & 0.4798 & 0.8820 & 0.4232 \\
\hline & CGS-LDA & \multirow{7}{*}{100} & 257.2486 & 0.3913 & 0.4770 & 0.1866 \\
\hline & ProdLDA & & 118.1967 & 0.3847 & 0.7850 & 0.3020 \\
\hline & ETM & & 56.5193 & 0.4210 & $\underline{0.9020}$ & 0.3798 \\
\hline & Hard VQ-TM & & 25.3745 & 0.4521 & 0.8280 & 0.3744 \\
\hline & Soft VQ-TM & & 25.8050 & 0.4550 & 0.8100 & 0.3685 \\
\hline & Multi-View Soft VQ-TM (\#Views=2) & & 13.1951 & $\underline{0.5045}$ & 0.8190 & $\underline{0.4132}$ \\
\hline & Multi-View Soft VQ-TM (\#Views=8) & & $\mathbf{1 1 . 9 4 7 8}$ & 0.4948 & 0.7470 & 0.3696 \\
\hline
\end{tabular}

Lower PPL is better, higher TC is better, higher TD is better, and higher TI is better. 
Table 2. Twenty Latent Topics Identified by CGS-LDA and ETM from $20 \mathrm{Ng}$

\begin{tabular}{|c|c|c|c|}
\hline Model & Topic ID & $\mathrm{TC}\left(C_{V}\right)$ & Top 10 Nearest Words \\
\hline \multirow{20}{*}{ CGS-LDA } & 0 & 0.4637 & [god, christian, jesus, people, bible, church, life, religion, faith, man] \\
\hline & 1 & 0.3256 & [file, entry, program, line, output, section, set, return, error, read] \\
\hline & 2 & 0.3711 & $\begin{array}{l}\text { [university, fax, phone, write, internet, center, research, computer, article, } \\
\text { information] }\end{array}$ \\
\hline & 3 & 0.3523 & [time, back, bike, write, thing, make, dod, ride, turn, leave] \\
\hline & 4 & 0.3255 & [power, ground, wire, work, water, current, circuit, make, sound, run] \\
\hline & 5 & 0.3241 & [number, write, point, article, time, rate, bit, give, man, difference] \\
\hline & 6 & 0.3311 & [problem, article, people, food, drug, write, study, year, time, effect] \\
\hline & 7 & 0.3706 & [gun, law, people, state, government, weapon, crime, police, kill, case] \\
\hline & 8 & 0.3775 & [drive, card, disk, system, problem, driver, mac, work, memory, dos] \\
\hline & 9 & 0.4466 & [people, turkish, armenian, armenians, woman, kill, child, turkey, armenia, turks] \\
\hline & 10 & 0.3374 & [question, make, argument, exist, claim, write, people, point, evidence, thing] \\
\hline & 11 & 0.4689 & [israel, war, jews, israeli, state, write, article, people, country, world] \\
\hline & 12 & 0.3469 & [president, make, work, money, people, tax, year, job, pay, clinton] \\
\hline & 13 & 0.5919 & [game, team, play, year, player, win, season, hockey, score, league] \\
\hline & 14 & 0.3798 & $\begin{array}{l}\text { [key, chip, encryption, system, government, clipper, security, privacy, phone, } \\
\text { secure] }\end{array}$ \\
\hline & 15 & 0.3874 & [car, buy, price, sell, good, sale, offer, drive, pay, engine] \\
\hline & 16 & 0.3297 & [post, send, list, book, group, information, mail, address, include, copy] \\
\hline & 17 & 0.4167 & $\begin{array}{l}\text { [window, file, image, program, run, display, version, application, server, } \\
\text { software] }\end{array}$ \\
\hline & 18 & 0.3323 & [write, article, people, thing, make, hear, read, good, post, opinion] \\
\hline & 19 & 0.4559 & [space, launch, nasa, system, earth, satellite, year, orbit, project, mission] \\
\hline \multirow{20}{*}{ ETM } & 0 & 0.3489 & [get, know, one, say, think, like, see, thing, people, time] \\
\hline & 1 & 0.3254 & [use, may, make, case, many, also, part, however, system, president] \\
\hline & 2 & 0.4505 & [god, jesus, christian, say, believe, bible, one, christ, make, belief] \\
\hline & 3 & 0.3682 & [gun, people, child, kill, drug, crime, weapon, police, case, claim] \\
\hline & 4 & 0.5255 & [datum, space, db, launch, output, hus, widget, dod, nasa, sun] \\
\hline & 5 & 0.3390 & [file, use, program, send, available, list, code, email, please, line] \\
\hline & 6 & 0.4139 & [hockey, team, new, division, san, canada, nhl, toronto, york, gm] \\
\hline & 7 & 0.3439 & [new, look, buy, price, good, sell, include, package, offer, like] \\
\hline & 8 & 0.3851 & [car, power, use, drive, speed, engine, wire, water, fast, low] \\
\hline & 9 & 0.4651 & [game, year, play, win, team, player, season, go, good, run] \\
\hline & 10 & 0.3312 & [information, make, get, please, mail, use, go, file, help, take] \\
\hline & 11 & 0.3378 & [book, first, one, time, science, study, earth, author, history, find] \\
\hline & 12 & 0.3516 & $\begin{array}{l}\text { [university, group, internet, information, computer, fax, center, year, call, } \\
\text { research] }\end{array}$ \\
\hline & 13 & 0.3628 & [thanks, david, john, appreciate, steve, mark, wonder, jim, mike, michael] \\
\hline & 14 & 0.3749 & [write, article, post, question, read, opinion, ask, please, yes, answer] \\
\hline & 15 & 0.3397 & [period, la, pt, vs, de, van, pp, cal, power, second] \\
\hline & 16 & 0.3607 & [key, government, law, use, encryption, state, chip, public, right, security] \\
\hline & 17 & 0.3354 & [go, take, back, one, day, put, get, right, also, call] \\
\hline & 18 & 0.3987 & [use, drive, system, window, card, run, windows, disk, problem, image] \\
\hline & 19 & 0.4854 & $\begin{array}{l}\text { [israel, people, war, israeli, jews, turkish, armenians, country, armenian, } \\
\text { government] }\end{array}$ \\
\hline
\end{tabular}


Table 3. Twenty Latent Topics Identified by Hard VQ-TM, Soft VQ-TM, and Multi-View Soft VQ-TMs from $20 \mathrm{Ng}$

\begin{tabular}{|c|c|c|c|}
\hline Model & Topic ID & TC $\left(C_{V}\right)$ & Top 10 Nearest Words \\
\hline \multirow{20}{*}{ Hard VQ-TM } & 0 & 0.3978 & [murder, law, criminal, child, death, court, police, case, woman, man] \\
\hline & 1 & 0.3923 & $\begin{array}{l}\text { [article, page, journal, magazine, constitution, newspaper, editor, report, } \\
\text { book, news] }\end{array}$ \\
\hline & 2 & 0.3738 & [really, think, know, want, something, get, thing, feel, lot, anything] \\
\hline & 3 & 0.3290 & [ball, back, right, water, inside, away, around, space, front, small] \\
\hline & 4 & 0.6077 & $\begin{array}{l}\text { [god, christ, jesus, christianity, christian, religious, religion, holy, church, } \\
\text { faith] }\end{array}$ \\
\hline & 5 & 0.3495 & [help, want, make, need, try, take, get, must, able, give] \\
\hline & 6 & 0.4231 & [john, mike, david, steve, michael, james, chris, tom, jim, scott] \\
\hline & 7 & 0.4001 & [write, read, publish, book, writer, please, reader, mail, page, author] \\
\hline & 8 & 0.6378 & $\begin{array}{l}\text { [software, pc, computer, server, disk, macintosh, windows, processor, } \\
\text { interface] }\end{array}$ \\
\hline & 9 & 0.5482 & [car, vehicle, engine, driver, truck, bus, wheel, motor, drive, speed] \\
\hline & 10 & 0.6024 & [ftp, unix, usenet, mb, newsgroup, pgp, server, toolkit, modem, faq] \\
\hline & 11 & 0.3340 & [think, might, need, much, whether, want, even, something, really, anything] \\
\hline & 12 & 0.3808 & [please, ask, call, tell, hear, want, answer, listen, know, let] \\
\hline & 13 & 0.4515 & $\begin{array}{l}\text { [use, method, technique, allow, equipment, manufacture, systems, tool, } \\
\text { available] }\end{array}$ \\
\hline & 14 & 0.6510 & [game, league, season, playoff, team, player, win, play, cup, hockey] \\
\hline & 15 & 0.4908 & $\begin{array}{l}\text { [mail, internet, phone, email, fax, information, telephone, service, access, } \\
\text { services] }\end{array}$ \\
\hline & 16 & 0.3348 & $\begin{array}{l}\text { [people, many, population, number, least, among, million, dead, muslims, } \\
\text { living] }\end{array}$ \\
\hline & 17 & 0.4787 & $\begin{array}{l}\text { [system, digital, software, systems, data, computer, use, video, standard, } \\
\text { format] }\end{array}$ \\
\hline & 18 & 0.4883 & $\begin{array}{l}\text { [university, institute, science, research, college, education, program, } \\
\text { professor, school] }\end{array}$ \\
\hline & 19 & 0.3281 & [year, last, two, government, world, three, time, since, first, week] \\
\hline \multirow{12}{*}{ Soft VQ-TM } & 0 & 0.3843 & [blood, hus, cancer, disease, die, girl, knife, child, black, death] \\
\hline & 1 & 0.4225 & $\begin{array}{l}\text { [government, security, palestinian, military, israeli, israel, peace, troops, } \\
\text { president] }\end{array}$ \\
\hline & 2 & 0.3572 & [go, get, want, let, take, stay, ca, wait, see, come] \\
\hline & 3 & 0.5139 & [billion, percent, million, rate, price, increase, dollar, higher, cost, per] \\
\hline & 4 & 0.6077 & $\begin{array}{l}\text { [god, christ, jesus, religion, christianity, religious, christian, church, holy, } \\
\text { faith] }\end{array}$ \\
\hline & 5 & 0.3368 & [help, try, make, able, want, allow, need, buy, use, seek] \\
\hline & 6 & 0.3785 & [know, think, anybody, want, really, anyone, anything, say, tell, nobody] \\
\hline & 7 & 0.4687 & $\begin{array}{l}\text { [mail, email, fax, phone, telephone, internet, mailing, information, service, } \\
\text { message] }\end{array}$ \\
\hline & 8 & 0.6417 & $\begin{array}{l}\text { [software, pc, server, computer, macintosh, windows, microsoft, unix, } \\
\text { modem, disk] }\end{array}$ \\
\hline & 9 & 0.5159 & [car, vehicle, truck, engine, wheel, driver, drive, bus, road, motorcycle] \\
\hline & 10 & 0.4252 & [interface, format, digital, color, user, standard, use, system, device, mode] \\
\hline & 11 & 0.3570 & $\begin{array}{l}\text { [problem, specific, whether, question, possible, matter, serious, risk, certain, } \\
\text { evidence] }\end{array}$ \\
\hline
\end{tabular}


Table 3. Continued

\begin{tabular}{|c|c|c|c|}
\hline Model & Topic ID & $\mathrm{TC}\left(C_{V}\right)$ & Top 10 Nearest Words \\
\hline & 12 & 0.3730 & [write, please, read, publish, tell, ask, mail, book, listen, reader] \\
\hline & 13 & 0.4241 & [two, three, one, four, five, six, second, another, single, first] \\
\hline & 14 & 0.4231 & [john, mike, david, steve, michael, chris, tom, james, jim, scott] \\
\hline & 15 & 0.4758 & $\begin{array}{l}\text { [university, research, science, institute, education, program, technology, } \\
\text { engineering] }\end{array}$ \\
\hline & 16 & 0.3269 & [many, several, like, people, various, number, among, popular, large, world] \\
\hline & 17 & 0.4034 & $\begin{array}{l}\text { [article, magazine, page, journal, constitution, editor, newspaper, edition, } \\
\text { publish] }\end{array}$ \\
\hline & 18 & 0.6201 & [game, season, league, win, cup, playoff, match, team, play, final] \\
\hline & 19 & 0.3597 & [really, good, think, lot, pretty, get, something, thing, feel, know] \\
\hline \multirow{27}{*}{$\begin{array}{c}\text { Multi-View } \\
\text { Soft VQ-TM } \\
(\# \text { Views=2) }\end{array}$} & 0 & 0.3820 & $\begin{array}{l}\text { [leader, orbit, wing, soviet, position, spacecraft, azerbaijan, scientist, party, } \\
\text { post] }\end{array}$ \\
\hline & 1 & 0.3272 & [buy, cheaper, sell, choose, install, prefer, please, purchase, want, modify] \\
\hline & 2 & 0.5089 & [god, christ, spirit, heaven, soul, worship, prophet, holy, earth, jesus] \\
\hline & 3 & 0.6396 & [unix, server, os, macintosh, dos, windows, ftp, mac, software, amiga] \\
\hline & 4 & 0.3518 & [pretty, really, feeling, feel, bit, fun, good, everybody, lot, decent] \\
\hline & 5 & 0.3374 & $\begin{array}{l}\text { [response, respond, similar, manner, resolution, voice, reaction, signal, action, } \\
\text { violent] }\end{array}$ \\
\hline & 6 & 0.4422 & [radio, mhz, channel, station, hd, converter, mb, pm, format, christian] \\
\hline & 7 & 0.4878 & $\begin{array}{l}\text { [professor, university, institute, science, research, engineering, college, } \\
\text { scientist] }\end{array}$ \\
\hline & 8 & 0.4764 & $\begin{array}{l}\text { [electronics, manufacturer, nazi, firearm, manufacture, card, cryptography, } \\
\text { chip, pt] }\end{array}$ \\
\hline & 9 & 0.4764 & [central, east, bank, south, road, km, services, west, north, state] \\
\hline & 10 & 0.4610 & [mask, hitter, helmet, damn, kid, pitcher, baseball, joe, waco, red] \\
\hline & 11 & 0.3365 & $\begin{array}{l}\text { [nuclear, distribute, destroy, acquire, oil, manufacture, prevent, sell, remove, } \\
\text { steal] }\end{array}$ \\
\hline & 12 & 0.4196 & [ball, wings, window, puck, red, rear, front, wing, injury, tear] \\
\hline & 13 & 0.6803 & [scsi, vga, mb, motherboard, amiga, cpu, floppy, macintosh, windows, rom] \\
\hline & 14 & 0.3549 & $\begin{array}{l}\text { [file, obtain, documentation, relevant, valid, submit, appropriate, } \\
\text { information] }\end{array}$ \\
\hline & 15 & 0.3907 & $\begin{array}{l}\text { [theory, problem, element, question, method, principle, argument, case, } \\
\text { solution] }\end{array}$ \\
\hline & 16 & 0.4026 & [rate, price, percent, billion, rise, increase, dollar, estimate, index, tax] \\
\hline & 17 & 0.3334 & [four, five, three, six, two, seconds, ten, nearly, car, third] \\
\hline & 18 & 0.4949 & [highest, hole, signature, andor, score, winner, pin, encrypt, datum, voltage] \\
\hline & 19 & 0.6347 & [mov, btw, ftp, homosexual, sex, marry, marriage, christianity, uucp, sexual] \\
\hline & 0 & 0.5670 & [mb, homosexual, atheist, mg, cache, simm, valid, mhz, hitler, device] \\
\hline & 1 & 0.3279 & [kevin, mike, al, goal, director, brother, bush, think, son] \\
\hline & 2 & 0.3805 & $\begin{array}{l}\text { [frequency, mhz, budget, space, digital, research, technology, useful, operate, } \\
\text { domain] }\end{array}$ \\
\hline & 3 & 0.4839 & [fax, mail, please, email, reply, phone, comment, sorry, posting, mailing] \\
\hline & 4 & 0.3460 & $\begin{array}{l}\text { [index, atlanta, rate, department, percent, fax, billion, economic, chicago, } \\
\text { report] }\end{array}$ \\
\hline & 5 & 0.5937 & [oo, eus, batf, simm, koresh, wm, stanley, mit, dod, nec] \\
\hline & 6 & 0.4527 & $\begin{array}{l}\text { [constitution, lewis, lebanese, murder, gordon, insurance, british, waco, wm, } \\
\text { koresh] }\end{array}$ \\
\hline
\end{tabular}


Table 3. Continued

\begin{tabular}{|c|c|c|c|}
\hline Model & Topic ID & $\mathrm{TC}\left(C_{V}\right)$ & Top 10 Nearest Words \\
\hline \multirow{13}{*}{$\begin{array}{l}\text { Multi-View } \\
\text { Soft VQ-TM } \\
(\# \text { Views=8) }\end{array}$} & 7 & 0.3606 & [army, islamic, military, la, police, fire, heavy, palestinian, wing, tank] \\
\hline & 8 & 0.3449 & $\begin{array}{l}\text { [request, letter, food, secretary, case, congress, disease, director, judge, } \\
\text { animal] }\end{array}$ \\
\hline & 9 & 0.5344 & [scsi, rocket, spacecraft, hd, fuel, radar, ac, converter, station, motherboard] \\
\hline & 10 & 0.6278 & $\begin{array}{l}\text { [datum, buf, stephanopoulos, compression, pixel, vga, delete, bias, genocide, } \\
\text { encrypt] }\end{array}$ \\
\hline & 11 & 0.4720 & [datum, mw, turkey, islamic, firearm, armenians, turks, turkish, island, tank] \\
\hline & 12 & 0.3695 & [goal, best, run, win, try, game, ahead, good, award, drive] \\
\hline & 13 & 0.5676 & $\begin{array}{l}\text { [algorithm, binary, unix, server, interface, byte, configuration, os, cpu, } \\
\text { encryption] }\end{array}$ \\
\hline & 14 & 0.5825 & [usenet, uucp, printf, btw, cryptography, newsgroup, vga, xt, widget, xlib] \\
\hline & 15 & 0.3411 & $\begin{array}{l}\text { [relative, economic, former, significant, israel, family, male, lebanon, slave, } \\
\text { war] }\end{array}$ \\
\hline & 16 & 0.4289 & [audio, bill, coverage, digital, law, card, services, full, cover, deep] \\
\hline & 17 & 0.3409 & $\begin{array}{l}\text { [state, religious, town, local, police, texas, political, power, culture, } \\
\text { government] }\end{array}$ \\
\hline & 18 & 0.4240 & [sick, care, option, son, older, insurance, family, child, choose, mine] \\
\hline & 19 & 0.5686 & [calgary, oo, min, bay, beat, buffer, jumper, neighbor, toolkit, mi] \\
\hline
\end{tabular}

Test Document ID 77:

Wow, this guy seems to be out to prove something to his old team, Boston. Which Sweeney you ask...well, of course Bob Sweeney, the one that Boston let Buffalo get a hold of (they still have 2 Sweeneys which makes things slightly confusing). Game winner in OT in game 1, and another BIG goal (seconds after Fuhr made 3 point blank saves $\rightarrow>$ this is why Grant has 5 rings!!!) to put Buffalo ahead in the 3rd. Yes, Neely countered a minute later, but hadn't this course of Buffalo going ahead after being tied and shutting down another few great scoring opportunities, I think Boston would have notched their first win of the series. Well, the Sabres haven't made it to the end of this series yet, but I certainly feel they've got Boston right were they want them... actually, they've got them in a position that neither Buffalo nor Boston felt that would come about One more astronomical game by Fuhr a few more heroics by the rest of the team (this is a team sport afterall) and I think Borque, Neely Jounea (sp?) and Company are gonna be swingin new stick (Weather is perfect for golf season) real soon. I'm not gonna waiger anything on this, becase new stick (Weather is perfect for golf season) real soon. Im not gonna waiger anything on this, because ive seen some realy strange things happen in both pro and college hockey. Talkng about golf.... was that hockey swing, golf swing or baseball swing that Hawerchuck used in the last shot of the game that Khmylev deflected in for the BIG ONE? The whole OT (all 1 minute of it!) was a tesiment to Buffalo's ability to really be persistent and grind it out in the end (something they weren't necessarily in the regular season). The Sabres pushed hard and forced Borque to blatently take down Bodger in the opening seconds. I don't normally like penalties being called in such ulras Finally, the Sabres won a faceoff (they weren't that hot in this dept the rest of the game) when LaFontaine scooped at the puck 3 times. When Hawerchuck took his shot (quite a boomer, but Blue stopped this one) he took a few steps over to get his own rebound and slapped at it again, without setting it up. I didn't realize it went in until the announcer started screaming, "They score, THEY SCORE!!!". The best was seein LaFontaine jumping up and down, skating a little bit, jumping some more, and then skating over to Brad May who he jumped on

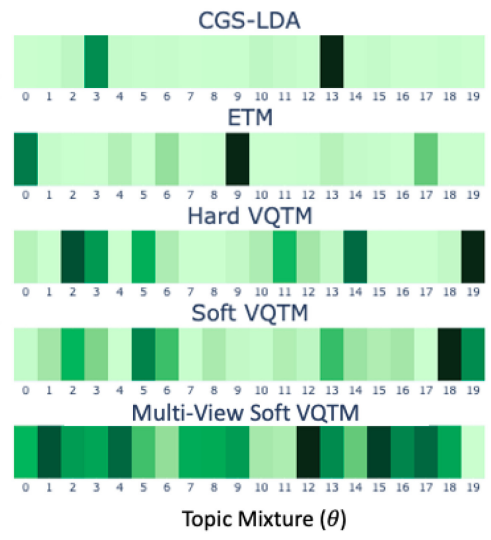

Fig. 6. Document-Topic mixture ( $\theta$ 's) inferred by all models for Test Document 77 from $20 \mathrm{Ng}$. (Darker color indicates higher probability.)

subtle topic mixtures, the VQ-TM family, particularly the Multi-View VQ-TM, excel in preserving the semantic richness of documents.

Now, in Figure 7, we display instantiation of topic-words association matrices computed by CGSLDA $\left(\beta_{c g s-l d a}\right), \operatorname{ETM}\left(\beta_{\text {etm }}\right)$, Hard VQ-TM $\left(\beta_{\text {hard-vqtm }}\right)$, Soft VQ-TM $\left(\beta_{\text {soft-vqtm }}\right)$, and Multi-View Soft VQ-TM ( $\left.\beta_{\text {multi-view-soft-vqtm }}\right)$, and all normalized with regard to vocabulary. Specifically, we demonstrate the interaction between 20 topics and a subset of $W_{\text {union }}$ :

$$
\begin{aligned}
W_{\text {union }}=\left(\bigcup_{i=0}^{K-1} W_{i_{C G S-L D A}}\right) & \circlearrowleft\left(\bigcup_{i=0}^{K-1} W_{i_{E T M}}\right) \bigcup\left(\bigcup_{i=0}^{K-1} W_{i_{\text {Hard-VQ-TM }}}\right), \\
& \circlearrowleft\left(\bigcup_{i=0}^{K-1} W_{i_{\text {Soft }-V Q-T M}}\right) \bigcup\left(\bigcup_{i=0}^{K-1} W_{i_{\text {Multi-View }- \text { Sof } t-V Q-T M}}\right),
\end{aligned}
$$

ACM Transactions on Intelligent Systems and Technology, Vol. 12, No. 3, Article 34. Publication date: April 2021. 


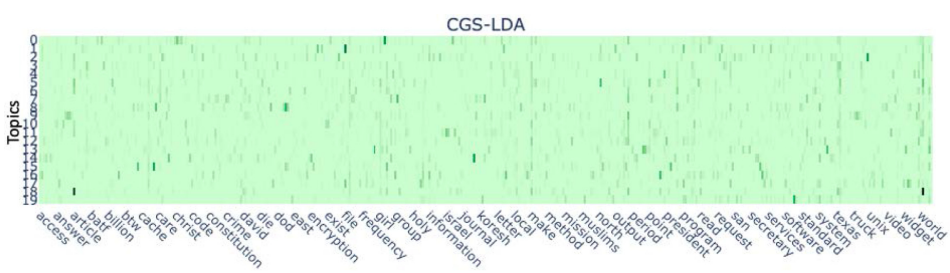

(a) Topic-Word association matrix $\left(\beta_{c g s-l d a}\right)$ by CGS-LDA

ETM

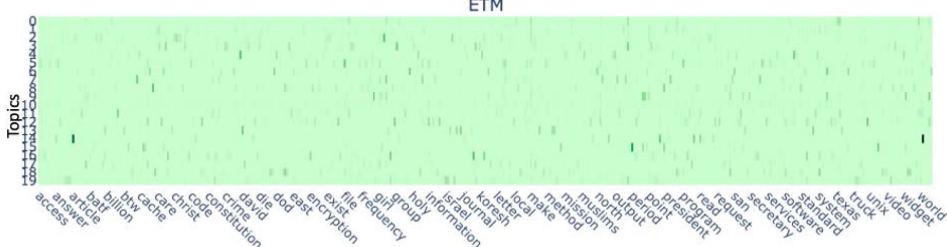

(b) Topic-Word association matrix $\left(\beta_{\text {etm }}\right)$ by ETM

Hard VQ-TM

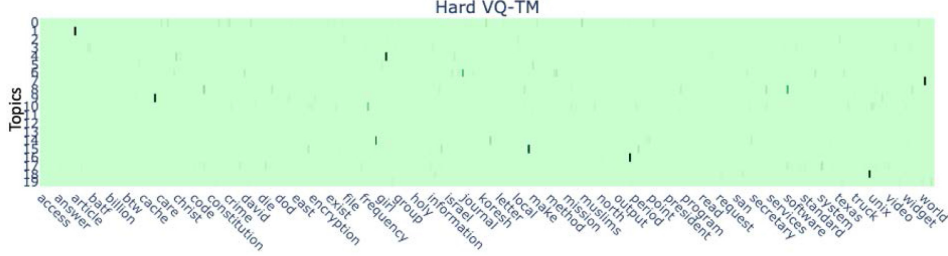

(c) Topic-Word association matrix $\left(\beta_{\text {hard-vqtm }}\right)$ by Hard VQ-TM Soft VQ-TM

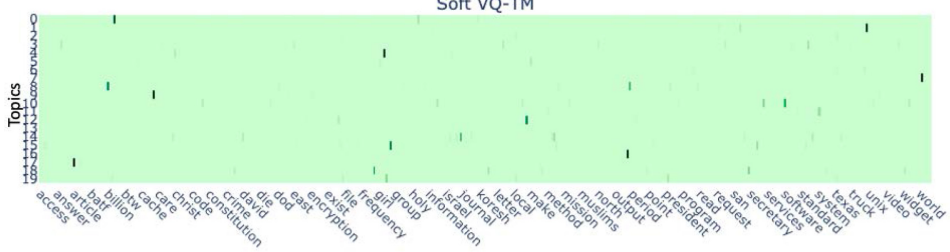

(d) Topic-Word association matrix $\left(\beta_{\text {soft-vqtm }}\right)$ by Soft VQ-TM

Multi-View Soft VQ-TM

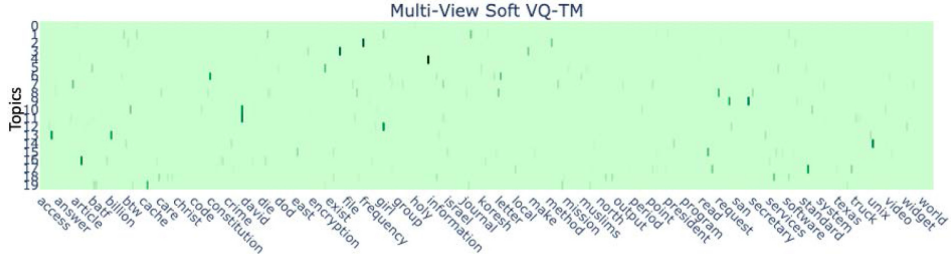

(e) Topic-Word association matrix $\left(\beta_{\text {multi-view-soft-vqtm }}\right)$ by Multi-View Soft VQ-TM(\#Views=8)

Fig. 7. Comparison of Topic-Word association matrices from $20 \mathrm{Ng}$.

(Darker color indicates higher probability.)

where

- $W_{i_{C G S-L D A}}, W_{i_{E T M}}, W_{i_{H a r d-V Q-T M}}, W_{i_{S o f t-V Q-T M}}$, and $W_{i_{\text {Multi-View-Soft-VQ-TM }}}$ represent the top C (10 in this work) words for the $i$-th topic from CGS-LDA, ETM, Hard VQ-TM, Soft VQ-TM, and Multi-View Soft VQ-TM models, respectively, and

- Wunion represents the union of all top words from all topics produced by all five models. ACM Transactions on Intelligent Systems and Technology, Vol. 12, No. 3, Article 34. Publication date: April 2021. 
It is clear from Figure 7 that the interaction between topics and words in all VQ-based topic models, especially Multi-View Soft VQ-TM, is much less uncertain as compared to baselines. This characteristic, coupled with the richness-preserving topic mixture, likely leads to the low PPL for VQ-TM models in document modeling.

5.1.2 Topic Coherence. With respect to TC (see Table 1), we observe that the VQ-TM model family supersedes other baselines. Let us gain further qualitative understanding of the model behavior by visualizing the relative positioning of topic and word embeddings in Figure 8. For example, referring to Tables 2 and 3, we choose Topic 8 (coherence $=0.3851$ ) in ETM results and Topic 9 (coherence $=0.5160)$ in Soft VQ-TM results, both of which cover the same notion of autos. By visualizing them and related words in Figure 8(b), we can see that the un-modified embeddings $\left(z_{e n}=e_{n}\right)$ of words such as road, engine, brake,and wheel are far from ETM Topic 8 . The Soft VQ-TM model transforms them into modified word embeddings $\left(z_{q n} \mathrm{~s}\right)$, which come much closer (depicted by arrows in Figure 8(b)) to Soft VQ-TM Topic 9 (autos). We attribute this phenomenon of member words converging toward topics to the attention vectors (characteristic of Soft VQ-TM; see Figure 4) and the $l_{v q}$ loss (characteristic of VQ-TM; see Equation (11)). We also witness the same pattern for other topics in Hard VQ-TM (Figure 8(a)) and Multi-View Soft VQ-TM (Figure 8(c)), which explains our VQ-TMs' strong performance on TC.

5.1.3 Topic Diversity and Topic Interpretability. In terms of TD (see Table 1), the Soft VQ-TM model leads on $20 \mathrm{Ng}$ but trails ETM on Conala; the Multi-View Soft VQ-TM seems to suffer considerably on the $20 \mathrm{Ng}$ corpus when number of topics is larger $(K=50$ or 100$)$. To understand this phenomenon, we reproduce a subset of $20 \mathrm{Ng}$ topics learned by Multi-View Soft VQ-TM $(K=50)$ in Table 4. We notice multiple occurences of the word nasa and canada. On one hand, there is indeed some level of redundancy with the canada-related topics, possibly due to the model struggling to partition overly fine granularity topics (after all, the natural number of topics is 20 ). On the other hand, some learned topics do convey subtly different themes. For example, of the two topics that share the word nasa, Topic 2 is related to science, whereas Topic 13 is more about organization. The word canada also appears in different themes such as government/politics, sports, and business/economics. This observation suggests that the TD metric, which totally relies on counting unique words (see Equation (20)), may have unfairly penalized the VQ-TM family's capability in dissecting subtly different themes. A systematic investigation of an alternative evaluation metric is outside the scope of the current study.

The product of topic coherence and diversity, topic interpretablity is affected by both. In our experiments (Table 1), VQ-TM models outperform baselines in most cases, with their strength in coherence outweighing the weakness in diversity.

\subsection{Discussion}

Based on the preceding analyses, we attribute the success of VQ-TMs to the following three distinguishing features:

(1) Leveraging richer input document representations: Instead of relying on BOW-based input representations as in all of the baseline models (CGS-LDA, ProdLDA, and ETM), we take advantage of bona fide distributed representations (word embeddings) and seamlessly integrate them into neural architectures.

(2) Learning discrete latent representations via VQ: In contrast to classical VAEs based on continuous latent representations, the discrete view of latent semantic space not only resonates perfectly with the mission of topic modeling but also avoids the posterior collapse issue. 


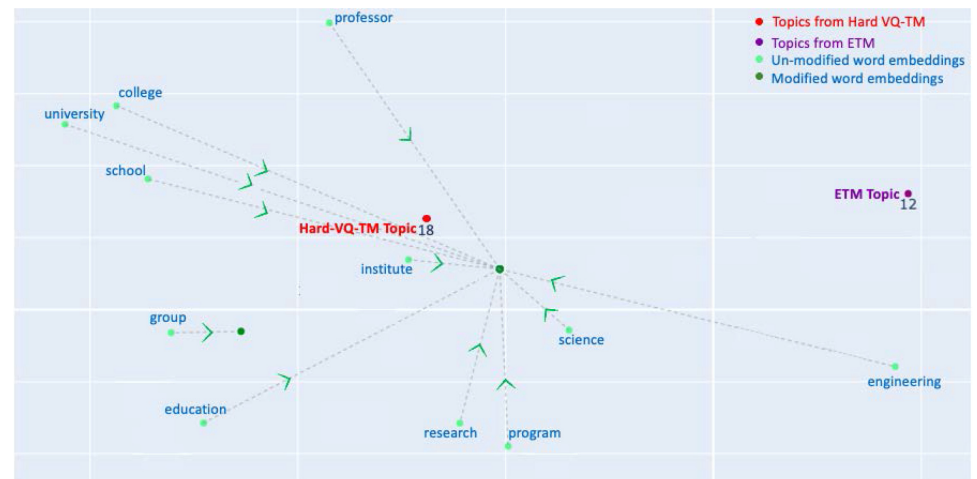

(a) Movement of words facilitated by Hard VQ-TM for Topic 18, which is identified as Topic 12 by ETM

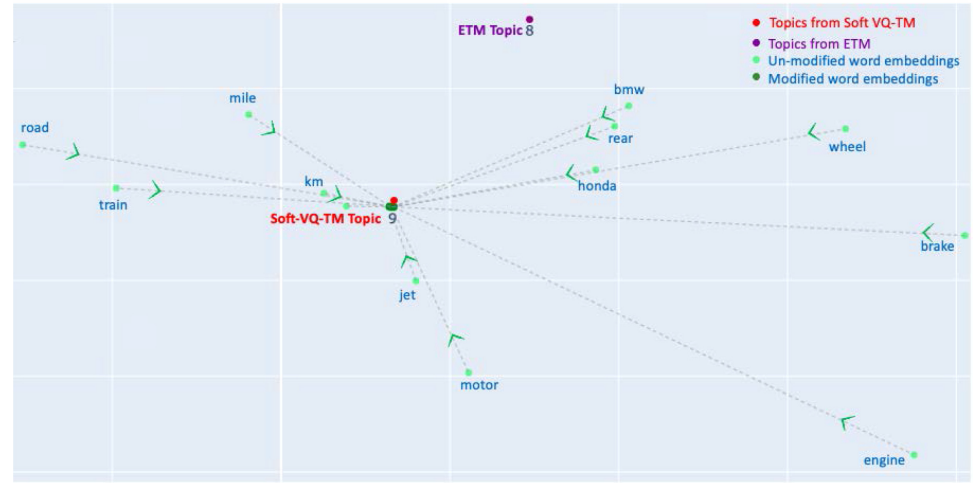

(b) Movement of words facilitated by Soft VQ-TM for Topic 9, which is identified as Topic 8 by ETM

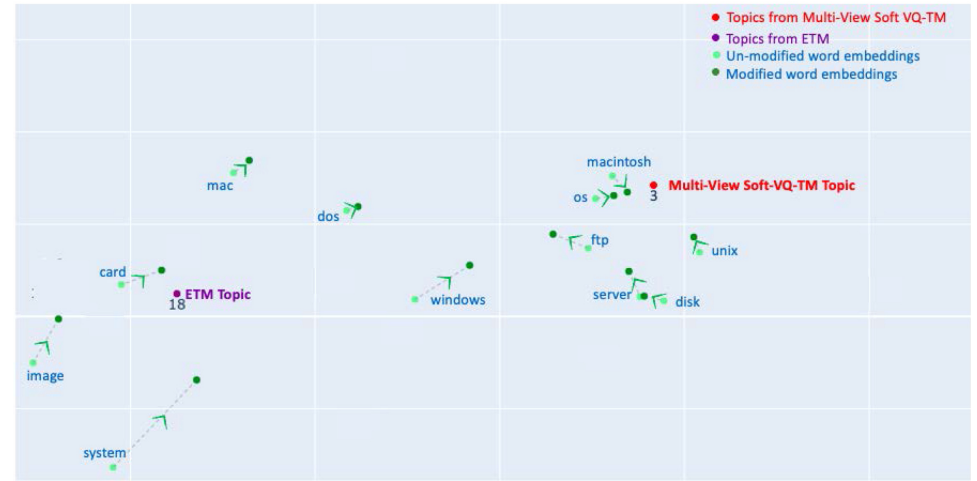

(c) Movement of words facilitated by Multi-View Soft VQ-TM (\#Views=2) for Topic 3, which is identified as Topic 18 by ETM

Fig. 8. Movement of words in various VQ-based topic models. (Arrow represents direction of movement.) 
Table 4. Subset of 50 Latent Topics Identified by Multi-View Soft VQ-TM from $20 \mathrm{Ng}$

\begin{tabular}{|c|c|c|c|}
\hline Model & Topic ID & $\mathrm{TC}\left(C_{V}\right)$ & Top 10 Nearest Words \\
\hline \multirow{30}{*}{$\begin{array}{l}\text { Multi-View } \\
\text { Soft VQ-TM } \\
(\# \text { Views }=2)\end{array}$} & 0 & 0.4983 & [mhz, cpu, mb, vga, iii, mw, seconds, circuit, st, motherboard] \\
\hline & 1 & 0.3374 & [gun, wire, cable, debate, fbi, newspaper, tax, gang, basis, plot] \\
\hline & 2 & 0.5110 & $\begin{array}{l}\text { [engineering, science, laboratory, research, university, nasa, technology, } \\
\text { physics] }\end{array}$ \\
\hline & $\vdots$ & $\vdots$ & \\
\hline & 13 & 0.3623 & $\begin{array}{l}\text { [nasa, injury, shuttle, staff, agency, investigation, manager, comment, } \\
\text { contract, accident] }\end{array}$ \\
\hline & : & : & : \\
\hline & 20 & 0.3926 & 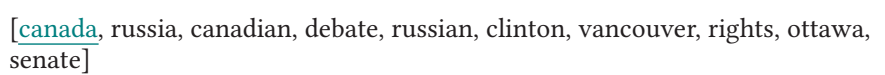 \\
\hline & 21 & 0.6826 & [usenet, newsgroup, hus, motherboard, cpu, mw, scsi, app, mb, modem] \\
\hline & 22 & 0.6572 & [hus, amiga, vga, atheist, pixel, bbs, newsgroup, buf, usenet, crypto] \\
\hline & 23 & 0.6296 & [toolkit, xt, pgp, andor, gif, vga, hus, faq, ftp, widget] \\
\hline & 24 & 0.4364 & $\begin{array}{l}\text { [canada, russia, canadian, russian, dollar, trade, soviet, vancouver, ottawa, } \\
\text { leafs] }\end{array}$ \\
\hline & 25 & 0.3960 & $\begin{array}{l}\text { [confuse, encrypt, remind, deserve, understanding, logic, understand, } \\
\text { acquire, appreciate] }\end{array}$ \\
\hline & 26 & 0.4444 & [canada, russia, hockey, nhl, canadian, vancouver, ice, sick] \\
\hline & 27 & 0.5166 & [summary, gmt, datum, session, ottawa, vs, motif, calgary, previous, apr] \\
\hline & 28 & 0.5315 & $\begin{array}{l}\text { [ottawa, hockey, vancouver, calgary, canada, nhl, leafs, debate, rights, } \\
\text { scientific] }\end{array}$ \\
\hline & 29 & 0.5473 & [km, lunar, calgary, mw, vancouver, datum, canada, ice, winnipeg, solar] \\
\hline & 30 & 0.3857 & $\begin{array}{l}\text { [canada, canadian, russia, western, trade, european, russian, region, } \\
\text { economic] }\end{array}$ \\
\hline & 31 & 0.4104 & $\begin{array}{l}\text { [canada, canadian, vancouver, russia, russian, ottawa, western, trade, } \\
\text { economic] }\end{array}$ \\
\hline & $\vdots$ & $\vdots$ & \\
\hline & 36 & 0.5234 & [summary, calgary, ottawa, gmt, nhl, leafs, vancouver, canada, session, round] \\
\hline & 37 & 0.5675 & [algorithm, sl, db, nec, apr, vs, rg, rf, fax, xt] \\
\hline & 38 & 0.6205 & [hockey, ice, vancouver, leafs, calgary, puck, winnipeg, canada, nhl, ottawa] \\
\hline & $\vdots$ & $\vdots$ & $\vdots$ \\
\hline & 41 & 0.5762 & $\begin{array}{l}\text { [canadian, canada, vancouver, ottawa, wiretap, calgary, scientist, quadra, } \\
\text { engineer] }\end{array}$ \\
\hline & $\vdots$ & $\vdots$ & $\vdots$ \\
\hline & 45 & 0.4783 & [canada, vancouver, canadian, nhl, russia, mine, dollar, ottawa, puck, leafs] \\
\hline & 46 & 0.5912 & [uucp, quadra, leafs, char, pit, russia, puck, hus, mine, orbit] \\
\hline & 47 & 0.4460 & [canada, canadian, hockey, russia, dollar, nhl, ice, vancouver, trade, spend] \\
\hline & 48 & 0.5282 & $\begin{array}{l}\text { [canada, canadian, ottawa, vancouver, calgary, hockey, nhl, soviet, russian, } \\
\text { russia] }\end{array}$ \\
\hline & 49 & 0.4909 & [convince, pray, puck, afraid, worry, remind, laugh, bother, encrypt, listen] \\
\hline
\end{tabular}




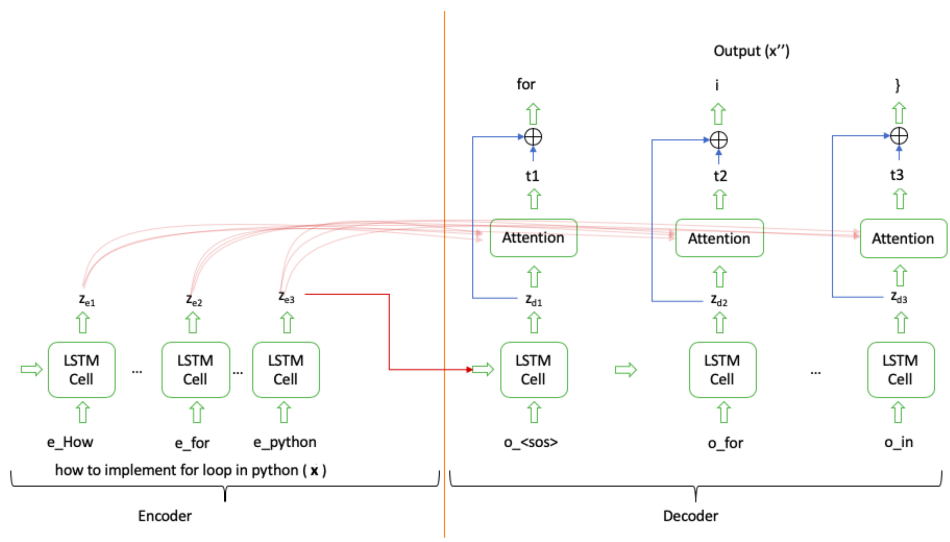

Fig. 9. Sequence-to-Sequence Model with Attention.

(3) Viewing words as mixtures of topics: This feature in Soft VQ-TM and Multi-View Soft VQTM is a major conceptual leap from the LDA family. By enabling direct, soft interaction between words and topics, our models explicitly model topic-level ambiguity of words and hence deliver superior performance in terms of PPL and TC.

Moving forward, in Section 6, we further illustrate the utility of VQ-TMs in a downstream task: code generation.

\section{CASE STUDY: VQ-TM-BASED CODE GENERATION}

Code generation is an interesting space where learning latent concepts (topics) may aid while generating a code snippet from an NL description. Neubig and Allamanis [26] define Coding as

$$
\text { Coding }=\text { Concept } \rightarrow \text { Implementation } .
$$

Highly impressive works such as those of Iyer et al. [18], Yin and Neubig [41], and Lin et al. [39] have capitalized on the ideas of neural machine translation (NMT), syntax of programming language, and semantic parsing, respectively. Yet as far as we know, our study is the first attempt to explicitly employ latent concepts while generating a code snippet as defined by Equation (23). We believe that high-level concepts such as the notion of variables, lists, and loops can be leveraged during the code generation process. It should be noted that this case study is for exploration purposes only. Specifically, it is meant to illustrate the feasibility of integrating topic embeddings into an autoregressive neural architecture and demonstrate the value added by learned latent concepts. There is no expectation of claiming state-of-the-art code generation results.

\subsection{Models}

The basic seq2seq model for NMT is first introduced by Sutskever et al. [35]. Then Iyer et al. [18] enhance NMT with attention (NMT-A) [1] for NL description generation. Here we use the same architectures as the backbone and baseline models for generating code snippets (Figure 9 presents an illustration of NMT-A). Specifically, for a given NL description $\left(n l \in U_{N L}\right)$, the aim is to produce $c^{*}$ that maximizes some scoring function $s \in\left(U_{C} \times U_{N L}\right) \rightarrow \mathbb{R}$ :

$$
c^{*}=\underset{c}{\operatorname{argmax}}(n l, c) \text {. }
$$

Here, $U_{N L}$ and $U_{C}$ represent the sets of NL descriptions and code snippets, respectively. We represent a code snippet $\left(\mathrm{c}=c_{1}, c_{2} \ldots c_{x}\right)$ as a sequence of one-hot vectors, $o_{c}=o_{c_{1}}, o_{c_{2}} \ldots o_{c_{x}} \in\{0,1\}^{N}$, 
where $N$ represents the vocabulary of code tokens. Formally, the following equations summarize the $g$-th decoding step:

$$
\begin{gathered}
s(c, n)=\prod_{g=1}^{x} p\left(c_{g} \mid c_{1}, c_{2} \ldots c_{g-1}\right), \\
p\left(c_{g} \mid c_{1}, c_{2} \ldots c_{g-1}\right) \propto W \tanh \left(W_{1} z_{d_{g}}+W_{2} t_{g}\right), \\
z_{d_{g}, m_{g}}=\mathrm{f}\left(o_{c_{g-1}}, z_{d_{g-1}}, m_{g-1} ; \theta\right), \\
t_{g}=\sum_{j=1}^{y} \alpha_{g, j} . z_{e_{j}}, \\
\alpha_{g, j}=\frac{\exp \left(z_{d_{g}} z_{e_{j}}^{T}\right)}{\sum_{j=1}^{y} \exp \left(z_{d_{g}} z_{e_{j}}^{T}\right)}
\end{gathered}
$$

where

- $\prod_{g=1}^{x} p\left(c_{g} \mid c_{1}, c_{2} \ldots c_{g-1}\right)$ represents the product of conditional next-word probabilities;

- $W \in \mathbb{R}^{N \times h_{g}}, W_{1} \in \mathbb{R}^{h_{g} \times h_{g}}$, and $W_{2} \in \mathbb{R}^{h_{g} \times h_{g}}$ are learned matrices, and $h_{g}$ is dimensionality of hidden state during decoding;

- $z_{d_{g}}$ and $m_{g}$ represents hidden state and cell state at the $g$-th decoding step;

- $f$ is computed by LSTM [17], which depends on one-hot representation $o_{c_{g-1}}$, hidden state $z_{d_{g-1}}$, and cell state $m_{g-1}$ of the previously decoded code token, $c_{g-1}$;

- $t_{g}$ is the contextual representation formulated by utilizing encoded hidden states, $z_{e_{j}} \mathrm{~s}$, of all words in NL description, $n l$, and normalized attention scores, $\alpha_{g, j}$ s; and

- $x$ and $y$ represent the length of code snippet and NL description, respectively.

While decoding, we utilize the beam search technique to find the optimal code snippet, $c^{*}$. Finally, during the training process, the negative log-likelihood of score $s$ is minimized to update the neural network parameters.

Intuitively, in NMT-A, any decoded token receives support from three sources:

(1) Weighted $\left(W_{1}\right)$ actual information present in $z_{d_{g}}$ (Equation (26)),

(2) Weighted $\left(W_{2}\right)$ contextual information present in $t_{g}$ (Equation (26)), and

(3) Beam search.

Inspired by our VQ-based topic models (see Section 3), we modify the NMT-A model by incorporating the notion of either (1) Hard VQ (HVQ), (2) Soft VQ (HVQ), or (3) Multi-View Soft VQ (MV-SVQ), as can be see in Figure 10.

Taking SVQ as an example, formally we replace Equation (26) with the following:

$$
\begin{gathered}
p\left(c_{g} \mid c_{1}, c_{2} \ldots c_{g-1}\right) \propto W \tanh \left(W_{1} z_{d_{g}}+W_{2} t_{g}+W_{3} z_{d q_{g}}\right), \\
u_{c_{g} t_{i}}=z_{d_{g}} \cdot E_{t_{i}}^{T}, \\
a_{c_{g} t_{i}}=\frac{\exp \left(u_{c_{g} t_{i}}\right)}{\sum_{i=0}^{K-1} \exp \left(u_{c_{g} t_{i}}\right)}, \\
z_{d q_{g}}=\sum_{i=0}^{K-1} a_{c_{g} t_{i}} * E_{t_{i}},
\end{gathered}
$$




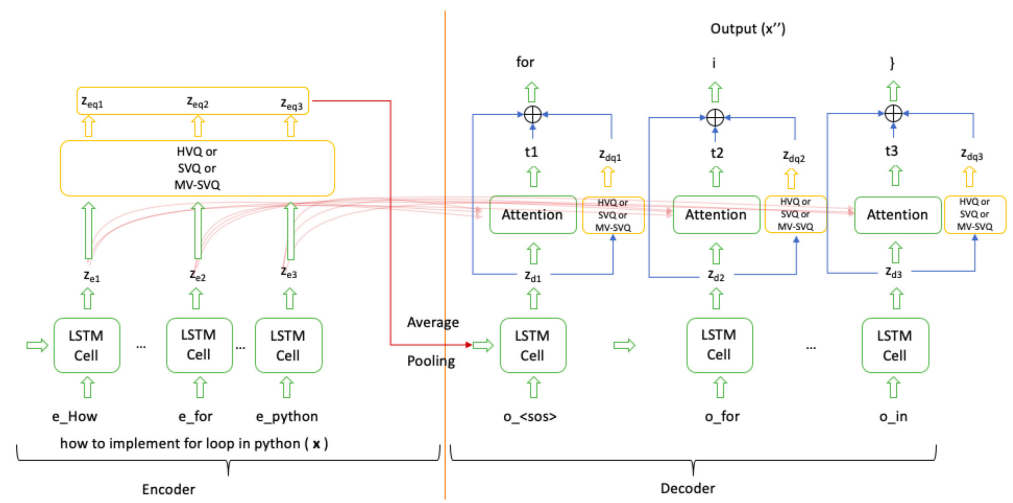

Fig. 10. Sequence-to-Sequence Model with Attention and VQ enhancement.

(HVQ corresponds to Hard VQ, SVQ refers to Soft VQ, and MV-SVQ is for Multi-View Soft VQ.)

where

- $W_{3} \in \mathbb{R}^{h_{g} \times h_{g}}$ represents a learned matrix;

- $z_{d q_{q}}$ is the quantized representation of decoded hidden state $z_{d_{g}}$ at the g-th step of the decoder;

- $E_{t_{i}}$ represents a concept embedding from LTS $\mathbf{E}$ that was pre-trained using Soft VQ-TM for the set of NL descriptions, $U_{N L}$.

Now, for a code token decoded at the $g$-th step, an extra source of support is available:

(4) Weighted $\left(W_{3}\right)$ conceptual information present in $z_{d q_{g}}$ (Equation (30)).

At the encoder end, we also incorporate the pre-trained LTS, E, to aid the decoding of the first code token at $g=0$. Formally,

$$
\begin{gathered}
u_{n l_{j} t_{i}}=z_{e_{j}} \cdot E_{t_{i}}^{T}, \\
a_{n l_{j} t_{i}}=\frac{\exp \left(u_{n l_{j} t_{i}}\right)}{\sum_{i=0}^{K-1} \exp \left(u_{n l_{j} t_{i}}\right)}, \\
z_{e q_{j}}=\sum_{i=0}^{K-1} a_{n l_{j} t_{i}} * E_{t_{i}}, \\
z_{e q}=\frac{\sum_{j=1}^{y} z_{e q_{j}}}{y} .
\end{gathered}
$$

Average pooled vector $z_{e q}$ is sent as a initial hidden state to the decoder. Last, we utilize the same loss function, negative log-likelihood of score $s$, as in the classical NMT-A.

\subsection{Training and Evaluation}

For all of the models, the hidden state of encoder and decoder has a dimension of 300; a learning rate of 0.001 is used. Moreover, pre-trained LTS, E, with $K=20$ and a beam search of beam size $=10$ are employed. The decoding procedure is halted when 10 tokens are generated. Again, a commitment cost ( $\gamma$ in $l_{v q}$ in Equation (11)) of 0.25 is implemented for any type of VQ-based NMT-A model. Finally, the Adam optimizer is used for training all models. 
Table 5. Results on the Conala Test Dataset for the Code Generation Task (Number of Pre-Trained Topics, $K=20$ )

\begin{tabular}{|c||c||c|}
\hline Model & $\begin{array}{c}\text { Corpus } \\
\text { BLEU-4 }\end{array}$ & $\begin{array}{c}\text { Mean Sentence } \\
\text { METEOR }\end{array}$ \\
\hline NMT & 0.90 & 17.81 \\
NMT-A & 5.90 & 19.37 \\
NMT-A-HVQ & 8.95 & 21.30 \\
NMT-A-SVQ & $\underline{\mathbf{9 . 8 1}}$ & $\underline{\mathbf{2 2 . 2 7}}$ \\
NMT-A-MV-SVQ & 9.26 & 21.70 \\
\hline
\end{tabular}

For evaluation, we report corpus-level BLEU-4 [29] and mean of sentence-level METEOR ${ }^{7}$ [2] scores. METEOR considers unigram overlaps between model-generated hypotheses and given references; it is a harmonic mean between unigram-precision and unigram-recall with more weight given to the latter. BLEU-4 depends on 4-gram precision (with uniform weights) and does not consider recall. To avoid a situation of 0 overlaps, we opt for a smoothing function where 1 is added to 0 counts when calculating the BLEU-4 score. Higher METEOR and BLEU-4 scores indicate better generation quality.

\subsection{Results and Discussion}

Quantitatively, we witness NMT-A with SVQ (NMT-A-SVQ) as a front-runner (Table 5). We also present the set of 20 topics learned by Soft VQ-TM from Conala in Table 6. The real significance of utilizing learned concept representations can be confirmed when a code snippet generated by a VQ-based NMT-A model is qualitatively more similar to the gold snippet as compared to NMTA. Hence, as an example, we conduct the practice of identifying concept(s) responsible for better generation at every timestep of decoding in NMT-A-SVQ.

In both test examples shown in Figure 11, the code snippets generated by the NMT-A-SVQ model exhibit better quality than those by NMT-A. Specifically, for example ID 1, the token id, acting as a variable in the gold code snippet, receives high support from Topic 12, which is related to variables and values. Similarly, Topic 6, related to integers, is the second most important concept while decoding the token integer. Moving on to example ID 2, we observe different concepts being utilized in the generation process. Specifically, the first token [ receives support from Topic 5, which is related to the data-structure list, and the second token remove_cruft is supported by Topic 4, which represents a concept of method. Our NMT-A-SVQ model shows impressive capability in leveraging high-level programming concepts to inform a simple sequential decoder. It is conceivable that the deployment of a stronger decoder (e.g., a syntax-guided tree decoder or a transformer-like architecture) may further improve code generation performance.

\section{CONCLUSION}

In this work, we direct our effort toward learning and utilizing explicit, dense topic embeddings in text corpora. In systematic empirical studies involving comprehensive evaluation metrics, the proposed variants of VQ-TMs exhibit superior performance compared to important baseline models, both quantitatively and qualitatively. In a case study on code generation, we successfully demonstrate the power of VQ-TMs by learning latent concepts in an autoencoding fashion but utilizing them in an autoregressive setting.

\footnotetext{
${ }^{7}$ Both are available at https://www.nltk.org/api/nltk.translate.html.
} 


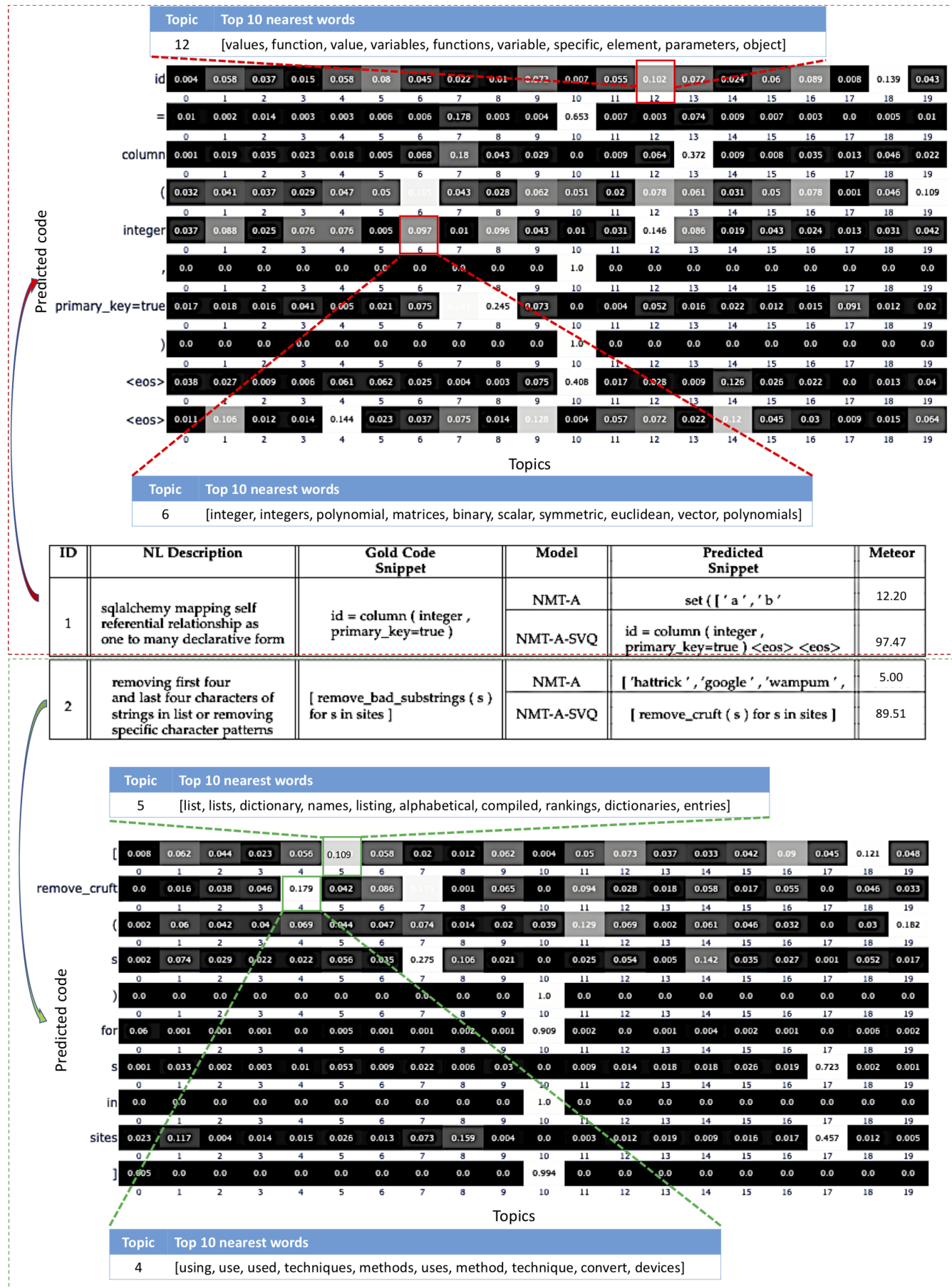

Fig. 11. Examples of Soft VQ-TM-based Code Generation (NMT-A-SVQ).

(Numbers in the matrices are token-to-topic attention scores.) 
Table 6. Twenty Latent Concepts Identified by Soft VQ-TM from Conala

\begin{tabular}{|c|c|c|c|}
\hline Model & Topic ID & $\mathbf{T C}\left(C_{V}\right)$ & Top 10 Nearest Words \\
\hline \multirow{20}{*}{ Soft VQ-TM } & 0 & 0.4568 & [conda, elif, ewm, dist, hangup, environ, doy, doen, vales, wagtail] \\
\hline & 1 & 0.3317 & [get, want, need, make, help, really, things, going, could, enough] \\
\hline & 2 & 0.5889 & [javascript, numpy, opengl, matlab, sql, php, gtk, debugger, jquery, mysql] \\
\hline & 3 & 0.4302 & $\begin{array}{l}\text { [encrypt, histogram, uncompressed, namespace, encrypting, decrypt, } \\
\text { debugging] }\end{array}$ \\
\hline & 4 & 0.3416 & $\begin{array}{l}\text { [using, use, used, techniques, methods, uses, method, technique, convert, } \\
\text { devices] }\end{array}$ \\
\hline & 5 & 0.2751 & $\begin{array}{l}\text { [list, lists, dictionary, names, listing, alphabetical, compiled, rankings, } \\
\text { dictionaries, entries] }\end{array}$ \\
\hline & 6 & 0.5809 & $\begin{array}{l}\text { [integer, integers, polynomial, matrices, binary, scalar, symmetric, euclidean, } \\
\text { vector] }\end{array}$ \\
\hline & 7 & 0.5784 & $\begin{array}{l}\text { [python, perl, php, javascript, pythons, scripting, opengl, bindings, lisp, } \\
\text { compiler] }\end{array}$ \\
\hline & 8 & 0.4151 & $\begin{array}{l}\text { [pandas, panda, orphaned, nesting, artificially, giant, chinese, eggs, spiders, } \\
\text { nest] }\end{array}$ \\
\hline & 9 & 0.3496 & $\begin{array}{l}\text { [array, multiple, arrays, different, various, types, images, shapes, objects, } \\
\text { sizes] }\end{array}$ \\
\hline & 10 & 0.4799 & [phong, sess, vincent, xavier, patricia, auc, died, elif, opened, queens] \\
\hline & 11 & 0.3558 & $\begin{array}{l}\text { [index, percent, prices, rate, output, average, indexes, composite, higher, } \\
\text { indicators] }\end{array}$ \\
\hline & 12 & 0.3657 & $\begin{array}{l}\text { [values, function, value, variables, functions, variable, specific, element, } \\
\text { parameters, object] }\end{array}$ \\
\hline & 13 & 0.6037 & [django, grappelli, iter, mozilla, ui, jquery, python, wagtail, gstreamer, lon] \\
\hline & 14 & 0.3514 & $\begin{array}{l}\text { [plot, plots, characters, character, plotting, subplots, movie, script, stories, } \\
\text { plotted] }\end{array}$ \\
\hline & 15 & 0.3530 & [two, three, four, five, second, first, one, third, last, year] \\
\hline & 16 & 0.5572 & [file, files, user, server, software, download, browser, data, folder, users] \\
\hline & 17 & 0.5081 & [vincent, patricia, sess, reorganize, auc, mapi, dosen, conda, died, hangup] \\
\hline & 18 & 0.5320 & $\begin{array}{l}\text { [string, strings, piano, violin, chords, stringed, instrument, keyboard, } \\
\text { concatenation, octave] }\end{array}$ \\
\hline & 19 & 0.3153 & [column, columns, writes, weekly, telegram, page, editor, mail, daily, e] \\
\hline
\end{tabular}

\section{APPENDIX}

\section{A VECTOR QUANTIZATION}

VQ [11] is a general form of pattern recognition that matches an input pattern against a stored set of latent codes. The technique involves a vector qunatizer that maps an input vector in $k$ dimensional Euclidean space, $\mathbb{R}^{\mathrm{k}}$, into a finite set $C$ (termed as codebook) containing $N(N<k)$ latent codes $y_{1}, y_{2} \ldots y_{N}$, where $y_{i} \in \mathbb{R}^{\mathrm{k}}$ and index set $i=\{1,2 . . N\}$.

A vector quantizer is, further, segregated into two components: vector encoder and vector decoder. Encoder $E$ maps (nearest-neighbor method) the input vector from $k$-dimensional space to one of the indices in set $i=\{1,2 . . N\}$. However, decoder $D$ outputs the vector $x^{\prime \prime}$ equal to latent code corresponding to the closest index from the set $C$. In summary, the overall vector-quantizer operation $\mathrm{Q}$ for an input $x$ can be regarded as

$$
\begin{gathered}
x^{\prime \prime}=Q(x)=D \cdot E(x)=D(E(x)) \\
E: R^{k} \rightarrow i \\
D: i \rightarrow R^{k} .
\end{gathered}
$$


To quantify the performance of the vector quantizer, a distortion measure $d$ between the input vector $x$ and the output quantized vector $x^{\prime \prime}$ is utilized. A good system yields a small average distortion. Although many alternatives exist, one of the most widely measures of distortion is squared error between two vectors defined as

$$
d\left(x, x^{\prime \prime}\right)=\sum_{j=1}^{k}\left(x_{j}-x_{j}^{\prime \prime}\right)^{2} .
$$

As an indication of accuracy achievable with the vector quantizer, code rate, $r$, is defined as follows:

$$
r=\frac{\log _{2} N}{k} .
$$

Code rate also measures the number of bits per vector component used to represent the vector input.

\section{REFERENCES}

[1] Dzmitry Bahdanau, Kyunghyun Cho, and Yoshua Bengio. 2015. Neural machine translation by jointly learning to align and translate. In Proceedings of the International Conference on Learning Representations (ICLR '15).

[2] Satanjeev Banerjee and Alon Lavie. 2005. METEOR: An automatic metric for MT evaluation with improved correlation with human judgments. In Proceedings of the ACL Workshop on Intrinsic and Extrinsic Evaluation Measures for Machine Translation and/or Summarization. 65-72.

[3] Bengio Yoshua, Courville Aaron, and Vincent Pascal. 2013. Representation learning: A review and new perspectives. IEEE Transactions on Pattern Analysis and Machine Intelligence 35 (2013), 1798-1828.

[4] David M. Blei and Jon D. Mcauliffe. 2008. Supervised topic models. In Advances in Neural Information Processing Systems 20. 121-128.

[5] David M. Blei, Andrew Y. Ng, and Michael I. Jordan. 2003. Latent Dirichlet allocation. fournal of Machine Learning Research 3 (2003), 993-1022.

[6] Samuel R. Bowman, Luke Vilnis, Oriol Vinyals, Andrew M. Dai, Rafal Jozefowicz, and Samy Bengio. 2016. Generating sentences from a continuous space. In Proceedings of the SIGNLL Conference on Computational Natural Language Learning.

[7] Scott Deerwester, Susan T. Dumais, George W. Furnas, Thomas K. Landauer, and Richard Harshman. 1988. Improving information retrieval with latent semantic indexing. In Proceedings of the Annual Meeting of the American Society for Information Science. 36-40.

[8] Paramveer S. Dhillon and Dean Foster. 2011. Multi-view learning of word embeddings via CCA. In Advances in Neural Information Processing Systems. 199-207.

[9] Adji B. Dieng, Francisco J. R. Ruiz, and David M. Blei. 2019. Topic Modeling in Embedding Spaces. Technical Report. Columbia University.

[10] Adji B. Dieng, Chong Wang, Jianfeng Gao, and John Paisley. 2016. TopicRNN: A recurrent neural network with long-range semantic dependency. In Proceedings of the International Conference on Learning Representations.

[11] Allen Gersho and Robert M. Gray. 1992. Vector Quantization and Signal Compression. Kluwer Academic.

[12] Robert M. Gray. 1984. Vector quantization. IEEE ASSP Magazine 1 (1984), 4-29. https://ieeexplore.ieee.org/stamp/ stamp.jsp?tp=\&arnumber $=1162229$

[13] Thomas L. Griffiths and Mark Steyvers. 2004. Finding scientific topics. Proceedings of the National Academy of Sciences 101 (2004), 5228-5235.

[14] Thomas L. Griffiths, Mark Steyvers, David M. Blei, and Joshua B. Tenenbaum. 2005. Integrating topics and syntax. In Advances in Neural Information Processing Systems 17. 537-544.

[15] Junxian He, Zhiting Hu, Berg Taylor Kirkpatrick, Ying Huang, and Eric P. Xing. 2017. Efficient correlated topic modeling with topic embedding. In Proceedings of the 23rd ACM SIGKDD International Conference on Knowledge Discovery and Data Mining. 225-233.

[16] I. Higgins, L. Matthey, A. Pal, C. Burgess, and X. Glorot. 2017. beta-VAE: Learning basic visual concepts with a constrained variational framework. In Proceedings of the International Conference on Learning Representations.

[17] Sepp Hochreiter and Jürgen Schmidhuber. 1997. Long short-term memory. Neural Computation 9 (1997), 1735-1780. 
[18] Srinivasan Iyer, Ioannis Konstas, Alvin Cheung, and Luke Zettlemoyer. 2016. Summarizing source code using a neural attention model. In Proceedings of the 54th Annual Meeting of the Association for Computational Linguistics (ACL'16). 2073-2083.

[19] Lang Ken. 1995. NewsWeeder: Learning to filter netnews. In Proceedings of the 12th International Conference on Machine Learning. 331-339.

[20] Diederik P. Kingma and Max Welling. 2013. Auto-encoding variational Bayes. arXiv:1312.6114

[21] Hugo Larochelle and Stanislas Lauly. 2012. A neural autoregressive topic model. In Advances in Neural Information Processing Systems. 2708-2716.

[22] Shaohua Li, Tat-Seng Chua, Jun Zhu, and Chunyan Miao. 2016. Generative topic embedding: A continuous representation of documents. In Proceedings of the 54th Annual Meeting of the Association for Computational Linguistics. 666-675.

[23] Shaohua Li, Jun Zhu, and Chunyan Miao. 2017. PSDVec: A toolbox for incremental and scalable word embedding. Neurocomputing 237 (2017), 405-409.

[24] Yang Liu, Zhiyuan Liu, Tat-Seng Chua, and Maosong Sun. 2015. Topical word embeddings. In Proceedings of the 29th AAAI Conference on Artificial Intelligence. 2418-2424.

[25] Yishu Miao, Lei Yu, and Phil Blunsom. 2016. Neural variational inference for text processing. In Proceedings of the International Conference on Machine Learning. 1727-1736.

[26] Graham Neubig and Miltiadis Allamanis. 2018. Modelling natural language, programs, and their intersection. In Proceedings of the 2018 Conference of the North American Chapter of the Association for Computational Linguistics: Tutorial Abstracts. 1-3.

[27] David Newman, Jey Han Lau, Karl Grieser, and Timothy Baldwin. 2010. Automatic evaluation of topic coherence. In Human Language Technologies: The 2010 Annual Conference of the North American Chapter of the Association for Computational Linguistics. 100-108.

[28] Alberto Paccanaro and Geoffrey E. Hinton. 2001. Learning distributed representations of concepts using linear relational embedding. IEEE Transactions on Knowledge and Data Engineering 13, 2 (2001), 232-244.

[29] Kishore Papineni, Salim Roukos, Todd Ward, and Wei-Jing Zhu. 2002. BLEU: A method for automatic evaluation of machine translation. In Proceedings of the 40th Annual Meeting of the Association for Computational Linguistics (ACL'02). 311-318.

[30] Jeffrey Pennington, Richard Socher, and Christopher Manning. 2014. Glove: Global vectors for word representation. In Proceedings of the 2014 Conference on Empirical Methods in Natural Language Processing (EMNLP'14). 1532-1543.

[31] James Petterson, Alex Smola, Tiberio Caetano, Wray Buntine, and Shravan Narayanamurthy. 2010. Word features for latent Dirichlet allocation. In Advances in Neural Information Processing Systems. 1921-1929.

[32] Michael Röder, Andreas Both, and Alexander Hinneburg. 2015. Exploring the space of topic coherence measures. In Proceedings of the International Conference on Web Search and Data Mining.

[33] Ruslan Salakhutdinov and Geoffrey Hinton. 2009. Replicated softmax: An undirected topic model. In Advances in Neural Information Processing Systems 22. 1607-1614.

[34] Akash Srivastava and Charles Sutton. 2017. Autoencoding variational inference for topic models. In Proceedings of the International Conference on Learning Representations.

[35] Ilya Sutskever, Oriol Vinyals, and Quoc V. Le. 2014. Sequence to sequence learning with neural networks. In Advances in Neural Information Processing Systems.

[36] Yee Whye Teh, Michael I. Jordan, Matthew J. Beal, and David M. Blei. 2006. Hierarchical Dirichlet processes. Journal of the American Statistical Association 101, 476 (2006), 1566-1581.

[37] Aaron van den Oord, Oriol Vinyals, and Koray Kavukcuoglu. 2017. Neural discrete representation learning. In Neural Information Processing Systems. 6306-6315.

[38] Ashish Vaswani, Noam Shazeer, Niki Parmar, Jakob Uszkoreit, Llion Jones, Aidan N. Gomez, Kaiser Lukasz, and Illia Polosukhin. 2017. Attention is all you need. In Advances in Neural Information Processing Systems, I. Guyon, U. V. Luxburg, S. Bengio, H. Wallach, R. Fergus, S. Vishwanathan, and R. Garnett (Eds.). Curran Associates, 5998-6008.

[39] Xi Victoria Lin, Chenglong Wang, Luke Zettlemoyer, and Michael D. Ernst. 2018. NL2Bash: A corpus and semantic parser for natural language interface to the Linux operating system. In Proceedings of the 11th International Conference on Language Resources and Evaluation.

[40] Pengcheng Yin, Bowen Deng, Edgar Chen, Bogdan Vasilescu, and Graham Neubig. 2018. Learning to mine aligned code and natural language pairs from stack overflow. In Proceedings of the International Conference on Mining Software Repositories. 476-486.

[41] Pengcheng Yin and Graham Neubig. 2017. A syntactic neural model for general-purpose code generation. In Proceedings of the 55th Annual Meeting of the Association for Computational Linguistics. 440-450.

Received November 2019; revised December 2020; accepted February 2021 